\title{
What explains gender inequalities in HIV/AIDS prevalence in sub-Saharan Africa? Evidence from the demographic and health surveys
}

Drissa Sia ${ }^{1 *}$ (D) Yentéma Onadja ${ }^{2}$, Mohammad Hajizadeh ${ }^{3}$, S. Jody Heymann ${ }^{4}$, Timothy F. Brewer ${ }^{5}$ and Arijit Nandi ${ }^{6}$

\begin{abstract}
Background: Women are disproportionally affected by human immunodeficiency virus (HIV)/acquired immunodeficiency syndrome (AIDS) in sub-Saharan Africa (SSA). The determinants of gender inequality in HIV/AIDS may vary across countries and require country-specific interventions to address them. This study aimed to identify the socio-demographic and behavioral characteristics underlying gender inequalities in HIV/AIDS in 21 SSA countries.

Methods: We applied an extension of the Blinder-Oaxaca decomposition approach to data from Demographic and Health Surveys and AIDS Indicator Surveys to quantify the differences in HIV/AIDS prevalence between women and men attributable to socio-demographic factors, sexual behaviours, and awareness of HIV/AIDS. We decomposed gender inequalities into two components: the percentage attributable to different levels of the risk factors between women and men (the "composition effect") and the percentage attributable to risk factors having differential effects on HIV/AIDS prevalence in women and men (the "response effect").

Results: Descriptive analyses showed that the difference between women and men in HIV/AIDS prevalence varied from a low of $0.68 \%(P=0.008)$ in Liberia to a high of $11.5 \%(P<0.001)$ in Swaziland. The decomposition analysis showed that $84 \%(P<0.001)$ and $92 \%(P<0.001)$ of the higher prevalence of HIV/AIDS among women in Uganda and Ghana, respectively, was explained by the different distributions of HIV/AIDS risk factors, particularly age at first sex between women and men. In the majority of countries, however, observed gender inequalities in HIV/AIDS were chiefly explained by differences in the responses to risk factors; the differential effects of age, marital status and occupation on prevalence of HIV/AIDS for women and men were among the significant contributors to this component. In Cameroon, Guinea, Malawi and Swaziland, a combination of the composition and response effects explained gender inequalities in HIV/AIDS prevalence.
\end{abstract}

Conclusions: The factors that explain gender inequality in HIV/AIDS in SSA vary by country, suggesting that country-specific interventions are needed. Unmeasured factors also contributed substantially to the difference in HIV/AIDS prevalence between women and men, highlighting the need for further study.

Keywords: Gender inequality, HIV/AIDS, Blinder-Oaxaca decomposition, Sub-Saharan Africa

\footnotetext{
* Correspondence: drissa.sia@uqo.ca

${ }^{1}$ Département des sciences infirmières, Campus de Saint-Jérôme, Université

du Québec en Outaouais, 5, rue Saint-Joseph, bureau J-3226, Saint Jérôme,

Québec J7Z 0B7, Canada

Full list of author information is available at the end of the article
} 


\section{Background}

Countries in sub-Saharan Africa (SSA) remain the most severely affected by the human immunodeficiency virus (HIV)/acquired immunodeficiency syndrome (AIDS) pandemic, accounting for $68 \%$ of all persons living with HIV/AIDS worldwide [1, 2]. Compared to men, women living in SSA are disproportionally affected by HIV/ AIDS, accounting for $59 \%$ of all infections in this region [1-3]. The gender disparity starts at a young age, with 15 to 24 year old women in SSA being more than twice as likely as men to become newly infected with $\operatorname{HIV}[1,2,4]$. There is an increasing recognition that prevention and treatment programs must address gender inequalities in HIV/AIDS [5]. Nevertheless, the mechanisms that give rise to these inequalities are poorly understood.

Gender inequalities in HIV/AIDS might be attributable to the differential distribution of risk factors for women and men. For example, the lower socioeconomic position of women in SSA may place them at greater behavioural risk for HIV infection [6-8]. Women are more likely to be uneducated, unemployed, and impoverished than men, which predisposes them to transactional sexual exchanges [9]. These sexual exchanges are often made with casual sex partners and without protection [10]. Thus, economic inequality between women and men may increase vulnerability to HIV among sexually active women [11]. Besides economic differences, unequal power relationships and the subordinate position of women relative to men also place women at higher risk for contracting HIV [12-18]. Women in SSA generally have less power to negotiate safe sex, including condom use [19]. Additionally, cultural factors encouraging older men-who are more likely to be HIV-infected-to have younger female partners (i.e., intergenerational sex) [20] limit women's ability to negotiate safe sex and increase the risk of HIV infection for women relative to men potentially exacerbating gender inequalities in HIV infection [21]. Moreover, social norms permitting violence against women, including domestic violence, spousal abuse, and rape might increase the probability of infection among women. This violence has many implications for the spread of HIV/AIDS. For example, it is associated with lack of condom use as well as traumatic injury among women in SSA [19], which increases risk of HIV infection [16, 22-25].

The differential responses of women and men to HIV/ AIDS risk factors may also contribute to observed gender inequalities in HIV/AIDS. For example, a recent study by Magadi [3] using pooled data from 20 SSA countries showed that conditioning on HIV risk factors, including sexual behaviors, did not explain the increased odds of HIV/AIDS among women relative to men [3], suggesting that traditional HIV risk factors may have differential and more detrimental effects for women compared to men.
Few studies [26, 27], however, have assessed whether risk factors have different effects on the probability of HIV/ AIDS for men and women. One study showed that unmarried women have twice the risk of HIV compared to unmarried men [28], suggesting that the differential effects of marital status may contribute to gender inequalities in HIV/AIDS. Additionally, although men and women may have similar distributions of household wealth, women have less control over household decision-making and financial resources and thereby may lack power to negotiate safe sexual practices (for example, condom use) with their partners $[9,29,30]$, which puts them at higher risk for HIV/AIDS. Unmeasured biological factors may also be important [31]. For example, male-to-female transmission of HIV is more biologically efficient than female-to-male transmission [32-35]. However, gender inequalities in HIV/AIDS vary substantially across world regions and are unlikely to be explained by biological differences alone. Political, organizational and legislative [5], social [12-18], and other cultural factors not already mentioned may also play important roles.

Gender inequalities in HIV/AIDS prevalence vary across countries [36]. Clarifying the determinants of gender inequalities in the SSA region, including whether they are explained by the differential distributions (a "composition effect") or effects (a "response effect") of HIV/AIDS risk factors for women and men, may help to inform countryspecific interventions for mitigating them [37, 38]. However, the characteristics explaining gender inequalities in HIV/AIDS prevalence in this region have not been systematically evaluated. Using data from the Demographic and Health Surveys (DHS) and AIDS indicator surveys (AIS), we recently elucidated the factors explaining gender inequalities in HIV/AIDS prevalence in Kenya, Lesotho and Tanzania [39]. This study showed that composition effects mainly explained gender inequalities in HIV/AIDS in Tanzania, whereas in Kenya and Lesotho they were partly explained by differences in the effects of measured HIV/ AIDS risk factors for men and women, including sociodemographic characteristics (i.e., age and marital status) and sexual behaviours (i.e., age at first sex). In the current study, we extended our previous work by: 1) measuring the magnitude of the gender inequality in HIV/AIDS prevalence across 21 SSA countries using available DHS; 2) quantifying the extent to which gender inequalities in HIV/AIDS were attributable to composition or response effects using a decomposition analysis; and 3) estimating the contribution of each risk factor to gender inequalities in HIV/AIDS prevalence across SSA countries.

\section{Methods}

Data

We used available data from the international DHS and the AIS to analyse the sources of gender inequality in 
HIV/AIDS prevalence across 21 SSA countries surveyed between 2003 and 2012 (Table 1). Each DHS is a crosssectional survey that collects and disseminates nationally representative household data, including comparable information on socio-demographic, behavioral, nutritional, health and other characteristics over time [22, 40, 41]. The DHS uses a multistage stratified design with probabilistic sampling that gives a defined probability of selection to each elementary unit [42]. Each DHS survey was stratified by urban and rural status and also by country-specific geographic or administrative regions [43]. To ensure comparability across countries and time, the DHS uses standardized measurement tools and techniques and an identical core questionnaire that is pretested and then administered by trained interviewers [44]. Further details concerning the DHS survey methodology are available elsewhere [45].

The AIS has been fielded in selected low- and middleincome countries since 2001 [3, 46]. Unlike sentinel surveillance, the AIS is a population-based survey that provides nationally-representative HIV prevalence data based on anonymous and voluntary testing of men and women aged 15-49 who were interviewed in the DHS, although some countries have also tested older adults [47, 48]. Due to the anonymous nature of the survey, respondents cannot be provided with their results. However, all respondents are offered referrals for free voluntary counselling and testing (VCT) and AIDS educational materials. In some countries, mobile VCT teams follow up after interviewers to counsel and test respondents who agree to be tested. The comparative nature of the DHS and the possibility to link HIV status from the AIS to the full DHS survey data, while conserving anonymity, provide a unique opportunity to examine factors contributing to gender inequalities in HIV/AIDS in different contexts in Africa. Data from the standard DHS linked to HIV prevalence data from the AIS were available for 313,207 respondents across 21 countries, with seven countries surveyed twice between 2003 and 2012.

We used secondary data collected by the international Demographic and Health Survey (DHS) program after obtaining participants' consent. Due to the anonymous nature of our data, our study was exempted from ethical committee review.

\section{Measures}

Our outcome of interest was HIV serostatus, determined by a confirmatory HIV-positive antibody blood serum result. Sex of the respondent (male vs. female, as defined in the DHS and AIS), used as a proxy for gender, was the key explanatory variable. Other covariates included socio-demographic characteristics, sexual behaviours, and HIV/AIDS awareness. Socio-demographic characteristics included urban/rural residence, the sex of the household head, the respondent's age at the time of survey, educational attainment (none, primary, or secondary and above), marital status (married, never married, or separated/divorced/widowed), and occupational type (agricultural, unemployed, domestic, trade, manual, office/service, or professional/manager). Applying a relative approach to poverty [49-51], household wealth was measured by a composite index created by principal component analysis (PCA) using information on household assets (ownership of radio, television, refrigerator, bicycle, motorcycle/ scooter, car/truck, and telephone), housing quality, and environmental conditions (electricity, source of drinking water, type of toilet facility, floor material). The wealth index was split into country-specific quintiles. Sexual behaviors included age at first marriage, age at first sex, premarital sex, sexual behavior risk (i.e., if a condom was not used at last sexual intercourse or having intercourse with a partner other than a spouse), and having multiple sex partners in the past year. Following the approach of Magadi [3], the PCA technique was employed to create a country-specific index of HIV/AIDS awareness using nine questions on knowledge of the modes of HIV transmission and ways to avoid infection.

\section{Statistical analysis}

We calculated the prevalence of HIV/AIDS for women and men across countries. The Chi-square test was used to estimate gender inequalities in HIV/AIDS as the difference in prevalence comparing women to men. Then, in countries where gender was significantly associated with HIV/AIDS prevalence, we explored the sources of gender inequalities in HIV/AIDS prevalence using an extension of the Blinder-Oaxaca (BO) decomposition $[52,53]$. This involved decomposing the observed women-men gaps in the prevalence of HIV/AIDS into two components: composition and response effects. Composition effects represent the contribution to gender inequalities in HIV/AIDS prevalence due to gender differences in the distributions of observable HIV/AIDS risk factors between women and men (i.e., socio-demographic characteristics, sexual behaviors, and HIV/AIDS awareness). Response effects reflect the contribution to gender inequalities in HIV/AIDS due to gender differences in the effects of measured HIV/AIDS risk factors, as well as unmeasured factors not included in the model [52-54]. The percentage of gender inequality in HIV/AIDS explained by a given component for each risk factor is defined by the amount of the difference in HIV/AIDS prevalence explained by the component divided by the total difference in HIV/AIDS prevalence between women and men multiplied by 100 . The BO method allowed us to assess which factors were associated with each source of inequality. Initially limited to continuous dependent variables, the $\mathrm{BO}$ decomposition approach has been extended 
Table 1 Response rates (\%), samples size and prevalence of HIV/AIDS (\%) by gender, country and survey year

\begin{tabular}{|c|c|c|c|c|c|c|c|c|c|c|c|c|}
\hline \multirow[b]{2}{*}{ Countries } & \multirow[t]{2}{*}{$\begin{array}{l}\text { Survey } \\
\text { year }\end{array}$} & \multicolumn{2}{|c|}{$\begin{array}{l}\text { Age range } \\
\text { (years) }\end{array}$} & \multirow[t]{2}{*}{$\begin{array}{l}\text { Response } \\
\text { rates (\%) }\end{array}$} & \multicolumn{3}{|c|}{ Women } & \multicolumn{3}{|l|}{ Men } & \multicolumn{2}{|c|}{$\begin{array}{l}\text { Gender inequality } \\
\text { in HIV/AIDS } \\
\text { prevalence }\end{array}$} \\
\hline & & Women & Men & & $\overline{n^{b}}$ & $\mathrm{HIV}+{ }^{c}$ & $\begin{array}{l}\text { Prevalence 1 } \\
(\%)^{\mathrm{d}}\end{array}$ & $\overline{n^{b}}$ & $\mathrm{HIV}+{ }^{\mathrm{c}}$ & $\begin{array}{l}\text { Prevalence } \\
(\%)^{d}\end{array}$ & Women-Men & $p$-value \\
\hline \multirow[t]{2}{*}{ Burkina Faso (BF) } & 2003 & $15-49$ & $15-59$ & 89 & 4189 & 84 & 1.82 & 3341 & 59 & 1.95 & -0.13 & 0.713 \\
\hline & 2010 & $15-49$ & $15-59$ & 95 & 8346 & 100 & 1.18 & 7034 & 60 & 0.84 & 0.34 & 0.086 \\
\hline \multirow[t]{2}{*}{ Cameroon (CM) } & 2004 & $15-49$ & $15-59$ & 91.34 & 5154 & 349 & 6.63 & 5041 & 203 & 3.92 & 2.71 & $<0.001$ \\
\hline & 2011 & $15-49$ & $15-59$ & 93 & 7253 & 434 & 5.57 & 6945 & 215 & 2.89 & 2.69 & $<0.001$ \\
\hline Congo Brazzaville (CG) & 2009 & $15-49$ & $15-49$ & 97.6 & 6349 & 240 & 4.13 & 5760 & 134 & 2.06 & 2.07 & $<0.001$ \\
\hline Côte d'Ivoire (Cl) & 2005 & $15-49$ & $15-49$ & 78 & 4547 & 247 & 6.21 & 3917 & 110 & 3.11 & 3.1 & $<0.001$ \\
\hline \multirow[t]{2}{*}{ Ethiopia (ET) } & 2005 & $15-49$ & $15-59$ & 79 & 5942 & 142 & 1.86 & 5107 & 70 & 0.91 & 0.95 & 0.003 \\
\hline & 2011 & $15-49$ & $15-59$ & 85.75 & 15505 & 358 & 1.86 & 12998 & 182 & 0.98 & 0.88 & $<0.001$ \\
\hline Ghana (GH) & 2003 & $15-49$ & $15-59$ & 85 & 5289 & 138 & 2.71 & 4265 & 68 & 1.63 & 1.08 & $<0.001$ \\
\hline Guinea (GN) & 2005 & $15-49$ & $15-59$ & 91 & 3842 & 68 & 1.89 & 2925 & 35 & 1.1 & 0.79 & 0.010 \\
\hline Liberia (LR) & 2005 & $15-49$ & $15-49$ & 84 & 6482 & 147 & 1.91 & 5206 & 62 & 1.23 & 0.68 & 0.008 \\
\hline \multirow[t]{2}{*}{ Malawi (MW) } & 2004 & $15-49$ & $15-54$ & 67 & 2864 & 421 & 13.32 & 2404 & 243 & 10.23 & 3.09 & 0.002 \\
\hline & 2010 & $15-49$ & $15-54$ & 87 & 7396 & 890 & 12.88 & 6509 & 530 & 8.39 & 4.5 & $<0.001$ \\
\hline Mali (ML) & 2006 & $15-49$ & $15-59$ & 88 & 4743 & 69 & 1.54 & 3886 & 38 & 1.11 & 0.44 & 0.109 \\
\hline Mozambique (MZ) & 2009 & $15-64$ & $15-64$ & 91 & 5901 & 875 & 12.67 & 4404 & 442 & 9.04 & 3.63 & $<0.001$ \\
\hline Niger (NE) & 2006 & $15-49$ & $15-59$ & 88 & 4441 & 39 & 0.71 & 3232 & 33 & 0.71 & 0 & 0.974 \\
\hline D.R. Congo (CD) & 2007 & $15-49$ & $15-59$ & 88 & 4632 & 81 & 1.62 & 4304 & 43 & 0.92 & 0.7 & 0.027 \\
\hline \multirow[t]{2}{*}{ Rwanda (RW) } & 2005 & $15-49$ & $15-59$ & 96.5 & 5663 & 222 & 3.61 & 4728 & 115 & 2.2 & 1.41 & $<0.001$ \\
\hline & 2010 & $15-49$ & $15-59$ & 98 & 6952 & 266 & 3.71 & 6296 & 154 & 2.41 & 1.3 & $<0.001$ \\
\hline $\begin{array}{l}\text { Sao Tome \& } \\
\text { Principe (ST) }\end{array}$ & 2008/2009 & $15-49$ & $15-59$ & - & 2550 & 37 & 1.29 & 2160 & 39 & 1.8 & -0.5 & 0.215 \\
\hline \multirow[t]{2}{*}{ Senegal (SN) } & 2005 & $15-49$ & $15-59$ & 80 & 4466 & 48 & 0.88 & 3250 & 16 & 0.44 & 0.44 & 0.009 \\
\hline & 2011 & $15-49$ & $15-59$ & 80 & 5590 & 61 & 0.83 & 4327 & 32 & 0.51 & 0.32 & 0.071 \\
\hline Sierra Leone (SL) & 2008 & $15-49$ & $15-59$ & 86 & 3466 & 64 & 1.73 & 3009 & 32 & 1.16 & 0.57 & 0.068 \\
\hline Swaziland (SZ) & $2006 / 2007$ & $15-49$ & $15-49$ & 85 & 4584 & 1438 & 31.15 & 3602 & 704 & 19.7 & 11.45 & $<0.001$ \\
\hline Uganda (UG) & 2011 & $15-59$ & $15-59$ & 96 & 11967 & 944 & 8.21 & 9399 & 551 & 6.11 & 2.1 & $<0.001$ \\
\hline Zambia (ZM) & 2007 & $15-49$ & $15-59$ & 75 & 5713 & 947 & 16.09 & 5161 & 649 & 12.29 & 3.8 & $<0.001$ \\
\hline \multirow[t]{2}{*}{ Zimbabwe (ZW) } & $2005 / 2006$ & $15-49$ & $15-54$ & 70 & 7494 & 1553 & 21.12 & 5555 & 782 & 14.75 & 6.37 & $<0.001$ \\
\hline & 2010/2011 & $15-49$ & $15-54$ & 75 & 7852 & 1463 & 17.71 & 6045 & 811 & 12.45 & 5.05 & $<0.001$ \\
\hline
\end{tabular}

Note: $n$ sample size

${ }^{a}$ More information on response rates is available at the following link: http://www.measuredhs.com/What-We-Do/survey-search.cfm?pgtype=main\&SrvyTp=country ${ }^{\mathrm{b}}$ Numbers of women and men in the sample. These frequencies are unweighted numbers

${ }^{c}$ Numbers of women and men with HIV positive test. These frequencies are unweighted numbers

${ }^{\mathrm{d}} \mathrm{A}$ Weighted percentage of persons with HIV positive test among women and men using sampling weights provided by the DHS and AIS

${ }^{e} p$-value based on Chi-squared test for the difference in HIV/AIDS prevalence between women and men

to the case of non-linear dependent variables [55-59]. Estimates were obtained using the statistical routine designed for non-linear outcomes described by Powers, Yoshioka and Yun [54]. This approach overcomes potential problems related to path dependence and identification [54]. All analyses, both descriptive and multivariate, were weighted using the available DHS sampling weights and accounted for clustering at the household level. We used STATA version 12 software for all analyses.

\section{Results}

\section{Gender inequalities in HIV/AIDS}

Table 1 reports response rates, samples size and prevalence of HIV/AIDS by gender, country and survey year. Women had a significantly higher prevalence of HIV/ AIDS than men in all countries and years sampled, apart from Burkina Faso in 2003 and 2010, Mali in 2006, Niger in 2006, Sao Tome \& Principe in 2008/09, Senegal in 2011 and Sierra Leone in 2011. The absolute difference 
between women and men in HIV/AIDS prevalence ranged from a low of $0.68 \%(P=0.008)$ in Liberia (2005) to a high of $11.5 \%(P<0.001)$ in Swaziland $(2006-7)$. Fig. 1 maps gender inequalities in HIV/AIDS prevalence in 21 SSA countries (using the most recent survey for countries with more than one available); inequalities were more pronounced in the southeastern region of SSA relative to the northwestern region.

\section{Sample characteristics}

Descriptive analyses (Additional file 1: Table S1) showed that there were differences in the distributions of HIV/ AIDS risk factors between women and men. On average, women were younger than men in all countries other than Mozambique, Swaziland, Liberia, Zimbabwe and Malawi, likely due to differences in the sampling frames for women and men, which ranged from 15 to 49 years for women and 15 to 64 years for men. In general, compared to men, women were more likely to be married (e.g., $76.7 \%$ versus $63.8 \%$ in Sierra Leone; $62.8 \%$ versus $50 \%$ in Cameroon) and to be separated/divorced/ widowed (e.g., $9.1 \%$ versus $5.4 \%$ in Ghana; $18.4 \%$ versus $5.5 \%$ in Mozambique). However, there were some exceptions. For example, in Malawi the percentages of married women and married men were statistically similar. There was no difference between the percentages of separated/divorced/widowed women and men in Mali. In general, women were more socioeconomically disadvantaged than men. For example, compared to men, fewer women had secondary or above education (e.g., $11.1 \%$ versus $31.9 \%$ in Guinea; $25.3 \%$ versus $36.6 \%$ in Uganda). Additionally, women were more likely than men to be unemployed or employed in trading, whereas men were more likely to be employed in professional/ managerial occupations. The descriptive statistics results also showed that a higher percentage of women reported

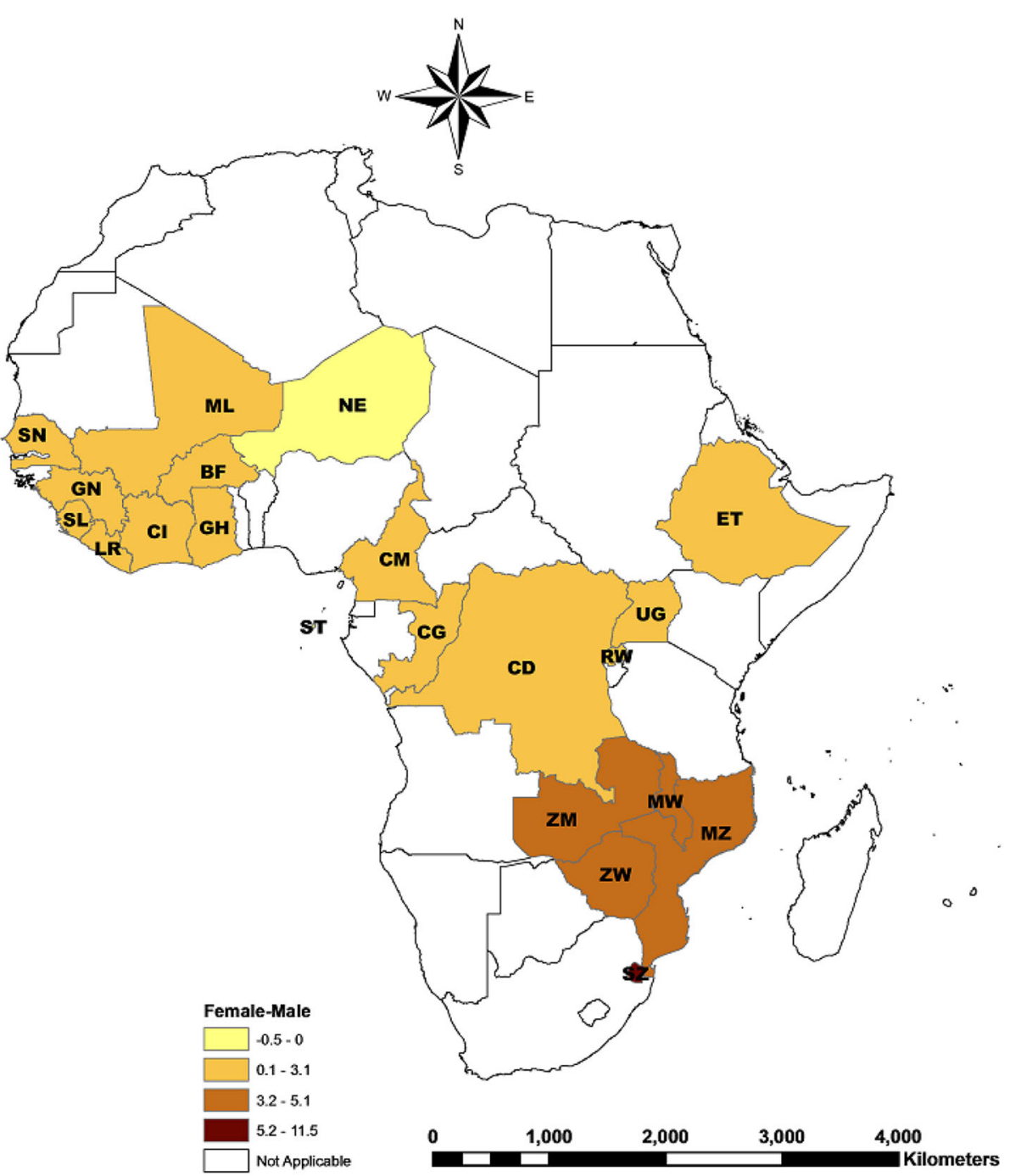

Fig. 1 Gender inequalities in HIV/AIDS prevalence in SSA countries 
first sexual intercourse before 16 years compared to men (e.g., $49.4 \%$ versus $19.4 \%$ in Guinea; $42.3 \%$ versus $27.8 \%$ in Côte d'Ivoire). With the exception of Swaziland, premarital sex was less common among women compared to men (e.g., $32.2 \%$ versus $65.9 \%$ in Malawi; $17.1 \%$ versus $41.8 \%$ in Rwanda). Women had lower levels of HIV/AIDS awareness compared to men, although this was not the case in all countries, for example in Swaziland.

\section{Explaining gender inequalities in HIV/AIDS prevalence}

We used the $\mathrm{BO}$ decomposition technique to examine sources of gender inequality in HIV/AIDS prevalence across countries (Table 2). There were three distinct patterns (Fig. 2). First, in the majority of countries, the response effect (the differential effect of a risk factor on women and men) explained the concentration of HIV/ AIDS among women; the percentage of the gender inequality in HIV/AIDS attributable to this component ranged from $81.5 \%$ in Mozambique and Rwanda to $116 \%$ in Congo Brazzaville. These results indicate that, had responses to HIV/AIDS risk factors been equivalent for men and women, the prevalence of HIV/AIDS would have been $19 \%$ lower among men relative to women in Mozambique and Rwanda and 16 \% higher among men relative to women in Congo Brazzaville. Second, in Uganda and Ghana, the composition effect (i.e., difference in distribution; the differential distribution of risk factors by gender) explained $84 \%$ and $92 \%$ of the higher prevalence of HIV/AIDS for women compared to men, respectively. Third, in Cameroon, Guinea, Malawi and Swaziland, both response and composition effects explained gender inequalities in HIV/AIDS prevalence. More than one-half of the gender inequality in HIV/AIDS prevalence in these countries was attributable to gender differences in responses to socio-demographic characteristics, sexual behaviors, HIV/AIDS awareness, and unmeasured risk factors.

Table 3 and Fig. 3 report detailed results of the decomposition analysis conducted within each country. Gender inequalities in the majority of countries were explained by the differential effects of socio-demographic factors, including age, marital status, and occupation, on prevalence of HIV/AIDS for women and men. Women aged 20-39 were more likely to be HIV-infected than men of the same age group, particularly in Congo Brazzaville (2009),

Table 2 Results from Blinder-Oaxaca decomposition analysis of gender inequalities in HIV/AIDS prevalence

\begin{tabular}{|c|c|c|c|c|c|c|c|c|}
\hline \multirow[t]{2}{*}{ Countries } & \multirow{2}{*}{$\begin{array}{l}\text { Survey } \\
\text { year }\end{array}$} & \multirow{2}{*}{$\begin{array}{l}\text { Gender inequality } \\
\text { in HIV/AIDS prevalence } \\
\text { (women-men) }\end{array}$} & \multicolumn{3}{|c|}{ Composition effect $^{f}$} & \multicolumn{3}{|c|}{ Response effect ${ }^{g}$} \\
\hline & & & Beta (SE) & $p$-value & Percent $^{d}$ & Beta (SE) & $p$-value & Percent $^{\mathrm{e}}$ \\
\hline Cameroon $^{c}$ & 2011 & 2.69 & $0.012(0.004)$ & 0.001 & 44.2 & $0.015(0.005)$ & 0.002 & 55.8 \\
\hline Congo Brazzaville & 2009 & 2.07 & $-0.003(0.005)$ & 0.478 & -15.6 & $0.024(0.007)$ & 0.000 & 115.6 \\
\hline Côte d'Ivoire ${ }^{a}$ & 2005 & 3.10 & $0.005(0.007)$ & 0.514 & 16.2 & $0.024(0.010)$ & 0.013 & 83.8 \\
\hline Ethiopia $^{a}$ & 2011 & 0.88 & $0.001(0.003)$ & 0.698 & 13.3 & $0.007(0.004)$ & 0.039 & 86.7 \\
\hline Ghana $^{\mathrm{b}}$ & 2003 & 1.08 & $0.01(0.003)$ & 0.000 & 91.9 & $0.001(0.004)$ & 0.811 & 8.1 \\
\hline Guinea $^{c}$ & 2005 & 0.79 & $-0.014(0.007)$ & 0.05 & -176.5 & $0.023(0.008)$ & 0.006 & 276.5 \\
\hline Liberia & 2007 & 0.68 & $-0.008(0.008)$ & 0.314 & -111.1 & $0.014(0.009)$ & 0.094 & 211.1 \\
\hline Malawi $^{c}$ & 2010 & 4.50 & $0.022(0.006)$ & 0.000 & 48.8 & $0.023(0.008)$ & 0.006 & 51.2 \\
\hline Mozambique $^{a}$ & 2009 & 3.63 & $0.006(0.011)$ & 0.583 & 18.5 & $0.026(0.013)$ & 0.051 & 81.5 \\
\hline D.R. Congo & 2007 & 0.70 & $-0.001(0.004)$ & 0.852 & -10.6 & $0.008(0.005)$ & 0.106 & 110.6 \\
\hline Rwanda ${ }^{a}$ & 2010 & 1.30 & $0.002(0.004)$ & 0.524 & 18.6 & $0.01(0.005)$ & 0.028 & 81.4 \\
\hline Swaziland ${ }^{c}$ & 2006/07 & 11.45 & $0.021(0.009)$ & 0.015 & 18.7 & $0.093(0.012)$ & 0.000 & 81.3 \\
\hline Uganda $^{\mathrm{b}}$ & 2011 & 2.10 & $0.018(0.003)$ & 0.000 & 83.7 & $0.003(0.005)$ & 0.476 & 16.3 \\
\hline Zambia $^{a}$ & 2007 & 3.80 & $0.005(0.008)$ & 0.522 & 13.9 & $0.031(0.01)$ & 0.003 & 86.1 \\
\hline Zimbabwe $^{a}$ & $2010 / 11$ & 5.05 & $0.009(0.007)$ & 0.188 & 17.7 & $0.044(0.010)$ & 0.000 & 82.3 \\
\hline
\end{tabular}

Note: using this method, the net percent contribution of both components always equals to 100. A contribution may be negative (less than zero) or positive and can even exceed 100. A positive contribution indicates that the component contributes to the greater prevalence of HIV/AIDS among women relative to men, whereas a negative contribution indicates the opposite

SE: Standard Error

${ }^{a}$ Countries where the difference between men and women in the response to risk factors mainly explains the gender gap at $p$-value $=5 \%$

${ }^{\mathrm{b}}$ Countries where the difference in the distribution of risk factors between men and women mainly explains the gender gap at $p$-value $=5 \%$

'Countries where difference in both the response to factors and the distribution of factors between men and women explains the gender gap at $p$-value $=5 \%$

dPart of gender inequality in HIV/AIDS prevalence attributable to differences in the distribution of risk factors

${ }^{\text {P }}$ art of gender inequality in HIV/AIDS prevalence attributable to differences in the effects of risk factors

${ }^{f}$ Represent the contribution to gender inequalities in HIV/AIDS prevalence due to gender differences in the distributions of observable HIV/AIDS risk factors between women and men

${ }^{9}$ Reflect the contribution to gender inequalities in HIV/AIDS due to gender differences in the effects of measured HIV/AIDS risk factors, as well as unmeasured factors not included in the model 


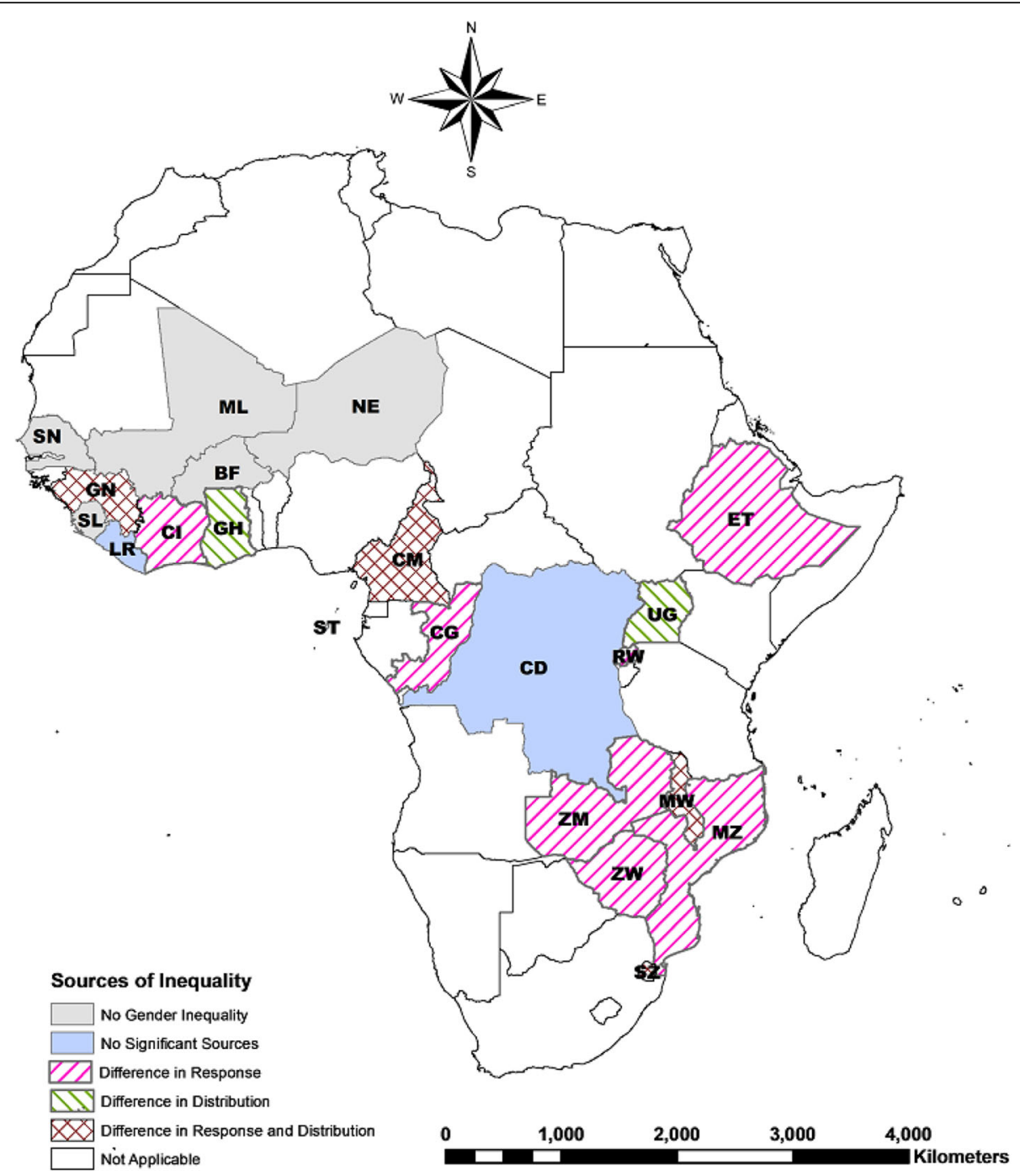

Fig. 2 Sources of gender inequalities in HIV/AIDS prevalence among SSA countries

Zambia (2007), and Zimbabwe (2010-11). The increased prevalence of HIV/AIDS among unmarried women compared to unmarried men explained $16.1 \%$ of the gender inequality in Côte d'Ivoire (2005), 43.1 \% in Rwanda (2010), 67.4 \% in Zambia (2007), and 75 \% in Zimbabwe (2010-11). Additionally, the differential effects of occupational status, particularly work in agriculture, contributed to higher HIV/AIDS prevalence for women compared to men in Côte d'Ivoire (68.1 \%), Ethiopia (50 \%), Mozambique (43.8 \%), and Rwanda (23.8\%). The constant term, which comprises the differential effects of factors not included in the model, was the largest contributor to gender inequalities in Congo Brazzaville.

In Ghana (2003) and Uganda (2011), it was the composition effect that explained gender inequalities in HIV/AIDS prevalence. Differences in the distribution of sexual behaviors explained $74.1 \%$ of the excess in HIV seropositivity among women in Ghana. For example, in
Ghana $24 \%$ of men reported that they had never had sex compared to $15.5 \%$ of women; results from the $\mathrm{BO}$ decomposition imply a $57.4 \%$ decrease in the gender inequality in HIV/AIDS if Ghanaian women and men were equally likely to report not having sex. In Uganda, the differential distribution of socio-demographic characteristics between women and men was responsible for $89 \%$ of the gender inequality in HIV/AIDS. The differential distributions of marital status, particularly being separated, divorced, or widowed, explained $38.6 \%$ and $21.9 \%$ of excess HIV/AIDS seropositivity among women in Ghana and Uganda, respectively. In both countries, the differential distributions of premarital sex between men (54.3\% in Ghana, $66 \%$ in Uganda) and women (43.1\% in Ghana, $45.5 \%$ in Uganda) reduced gender gaps in HIV/AIDS prevalence.

In countries where both response and composition effects explained gender inequalities in HIV/AIDS 
Table 3 Contribution of HIV/AIDS risk factors to gender inequalities in HIV/AIDS prevalence by country; decomposition analysis using latest available DHS, 2003 to 2011

\begin{tabular}{|c|c|c|c|c|c|c|c|c|}
\hline & $\begin{array}{l}\text { Ghana }^{a} \\
2003\end{array}$ & $\begin{array}{l}\text { Uganda } \\
2011\end{array}$ & $\begin{array}{l}\text { Congo Brazzaville } \\
2009\end{array}$ & $\begin{array}{l}\text { Côte d'Ivoire } \\
2005\end{array}$ & $\begin{array}{l}\text { Ethiopia }^{a} \\
2011\end{array}$ & $\begin{array}{l}\text { Rwanda } \\
2010\end{array}$ & $\begin{array}{l}\text { Zambia }^{\mathrm{a}} \\
2007\end{array}$ & $\begin{array}{l}\text { Zimbabwe } \\
\text { 2010/11 }\end{array}$ \\
\hline & \multicolumn{8}{|c|}{ Absolute contribution ${ }^{\mathrm{e}}$ to gender inequalities in HIV/AIDS prevalence ( $p$-value) } \\
\hline $\begin{array}{l}\text { Gender Difference in } \\
\text { HIV/AIDS Prevalence (\%) }\end{array}$ & 1.08 & 2.1 & 2.07 & 3.1 & 0.88 & 1.3 & 3.8 & 5.05 \\
\hline The composition effect ${ }^{\text {hg }}$ & $0.99(0.000)$ & $1.76(0.000)$ & $-0.32(0.478)$ & $0.5(0.514)$ & $0.12(0.698)$ & $0.24(0.524)$ & $0.53(0.480)$ & $0.89(0.188)$ \\
\hline $\begin{array}{l}\text { Socio-economic and } \\
\text { demographic characteristics }\end{array}$ & 0.160 & 1.870 & -0.320 & 0.750 & 0.160 & -1.500 & -2.44 & 0.830 \\
\hline Residence & 0.000 & 0.040 & 0.000 & -0.020 & 0.020 & 0.040 & 0.38 & 0.080 \\
\hline Urban & $0(0.86)$ & $0.02(0.031)$ & $0(0.924)$ & $-0.01(0.391)$ & $0.01(0.604)$ & $0.02(0.831)$ & $0.18(0.943)$ & $0.04(0.013)$ \\
\hline Rural & $0(0.86)$ & $0.02(0.031)$ & $0(0.924)$ & $-0.01(0.391)$ & $0.01(0.604)$ & $0.02(0.831)$ & $0.20(0.943)$ & $0.04(0.013)$ \\
\hline Sex of household head & 0.080 & 0.580 & 0.060 & -0.040 & 0.040 & -1.060 & -0.78 & 0.300 \\
\hline Male & $0.04(0.06)$ & $0.29(0.000)$ & $0.03(0.539)$ & $-0.02(0.538)$ & $0.02(0.654)$ & $-0.53(0.833)$ & $-0.33(0.943)$ & $0.15(0.168)$ \\
\hline Female & $0.04(0.06)$ & $0.29(0.000)$ & $0.03(0.539)$ & $-0.02(0.538)$ & $0.02(0.654)$ & $-0.53(0.833)$ & $-0.39(0.943)$ & $0.15(0.168)$ \\
\hline Age group & 0.040 & 0.110 & -0.130 & -0.120 & -0.010 & -0.020 & 0.14 & 0.490 \\
\hline $15-19$ & $0.02(0.059)$ & $0.06(0.001)$ & $-0.03(0.176)$ & $-0.07(0.297)$ & $-0.02(0.619)$ & $-0.05(0.825)$ & $0.15(0.943)$ & $0.42(0.000)$ \\
\hline $20-29$ & $0.01(0.409)$ & $0.06(0.026)$ & $0.01(0.738)$ & $0(0.985)$ & $0.01(0.644)$ & $0.01(0.894)$ & $-0.11(0.943)$ & $0.01(0.651)$ \\
\hline $30-39$ & $0.02(0.007)$ & $-0.02(0.000)$ & $-0.08(0.183)$ & $-0.03(0.288)$ & $0.01(0.616)$ & $-0.17(0.828)$ & $0.12(0.943)$ & $0.18(0.000)$ \\
\hline $40+$ & $-0.01(0.65)$ & $0.01(0.801)$ & $-0.03(0.208)$ & $-0.02(0.313)$ & $-0.01(0.64)$ & $0.19(0.819)$ & $-0.02(0.951)$ & $-0.12(0.002$ \\
\hline Education level & -0.030 & 0.330 & -0.040 & 0.030 & -0.040 & 0.030 & 0.12 & -0.100 \\
\hline None & $-0.02(0.14)$ & $0.08(0.182)$ & $-0.01(0.763)$ & $-0.01(0.931)$ & $-0.03(0.599)$ & $0.01(0.863)$ & $0.17(0.943)$ & $-0.06(0.029$ \\
\hline Primary & $0(0.24)$ & $0.02(0.011)$ & $0(0.983)$ & $0(0.548)$ & $-0.01(0.633)$ & $0(0.876)$ & $-0.20(0.943)$ & $0.12(0.053)$ \\
\hline Secondary and above & $-0.01(0.633)$ & $0.23(0.005)$ & $-0.03(0.674)$ & $0.04(0.68)$ & $0(0.683)$ & $0.02(0.848)$ & $0.15(0.943)$ & $-0.16(0.041)$ \\
\hline Standard of living & -0.010 & 0.030 & -0.050 & 0.030 & 0.000 & 0.130 & 0.28 & -0.050 \\
\hline 1st Quintile & $0(0.816)$ & $0(0.061)$ & $-0.02(0.257)$ & $0(0.365)$ & $0(0.641)$ & $0.09(0.828)$ & $0.10(0.943)$ & $0.02(0.186)$ \\
\hline 2nd Quintile & $0(0.626)$ & $0.02(0.071)$ & $0(0.52)$ & $-0.01(0.3)$ & $0(0.731)$ & $-0.01(0.873)$ & $-0.01(0.943)$ & $0(0.67)$ \\
\hline 3rd Quintile & $0(0.814)$ & $0.01(0.009)$ & $0(0.868)$ & $-0.01(0.626)$ & $0(0.725)$ & $0(0.973)$ & $0.09(0.943)$ & $0(0.64)$ \\
\hline 4th Quintile & $0(0.082)$ & $0(0.735)$ & $-0.01(0.3)$ & $0(0.406)$ & $0(0.642)$ & $0.03(0.834)$ & $0.10(0.943)$ & $-0.02(0.472$ \\
\hline 5th Quintile & $-0.01(0.144)$ & $0(0.41)$ & $-0.02(0.255)$ & $0.05(0.336)$ & $0(0.678)$ & $0.02(0.846)$ & $0(0.952)$ & $-0.05(0.04)$ \\
\hline Occupation type & 0.040 & -0.030 & - & 0.930 & 0.080 & 0.160 & -1.00 & -0.370 \\
\hline Unemployed & $0(0.706)$ & $-0.22(0.031)$ & - & $0.45(0.283)$ & $0.04(0.661)$ & $-0.11(0.85)$ & $-0.11(0.950)$ & $-0.02(0.919$ \\
\hline Agricultural & $0.03(0.143)$ & $0.14(0.209)$ & - & $-0.74(0.265)$ & $0.02(0.796)$ & $0.16(0.822)$ & $-0.97(0.943)$ & $-0.04(0.838$ \\
\hline Domestic & - & $0.02(0.167)$ & - & - & - & $0(0.872)$ & - & $0.03(0.263)$ \\
\hline Trade & $0(0.885)$ & $-0.01(0.79)$ & - & $1.22(0.291)$ & $0.01(0.705)$ & $-0.01(0.832)$ & $-1.12(0.940)$ & $-0.06(0.487)$ \\
\hline Manual labor & $0.02(0.069)$ & $0.05(0.233)$ & - & $-0.33(0.265)$ & $0(0.6)$ & $0.15(0.81)$ & $0.15(0.943)$ & $-0.32(0.196)$ \\
\hline Office/service & $0.01(0.276)$ & $0.02(0.57)$ & - & $0.02(0.287)$ & $0(0.669)$ & $-0.05(0.835)$ & $0.08(0.943)$ & $0.02(0.562)$ \\
\hline Professional/manager & $-0.02(0.221)$ & $-0.03(0.665)$ & - & $0.31(0.258)$ & $0.01(0.61)$ & $0.02(0.844)$ & $-0.03(0.943)$ & $0.02(0.57)$ \\
\hline Marital status & 0.040 & 0.810 & -0.160 & -0.060 & 0.070 & -0.780 & -1.58 & 0.480 \\
\hline Never married & $0.03(0.151)$ & $0.4(0.000)$ & $-0.08(0.438)$ & $0.03(0.732)$ & $0.04(0.617)$ & $-0.26(0.829)$ & $-0.90(0.943)$ & $0.38(0.154)$ \\
\hline Married & $0(0.986)$ & $-0.05(0.013)$ & $-0.12(0.18)$ & $-0.11(0.324)$ & $0(0.762)$ & $-0.03(0.832)$ & $0.17(0.943)$ & $-0.6(0.002)$ \\
\hline $\begin{array}{l}\text { Separated/divorced/ } \\
\text { widowed }\end{array}$ & $0.01(0.065)$ & $0.46(0.000)$ & $0.04(0.222)$ & $0.02(0.324)$ & $0.03(0.621)$ & $-0.49(0.829)$ & $-0.85(0.943)$ & $0.7(0.000)$ \\
\hline Sexual behavior factors ${ }^{f}$ & 0.800 & -0.080 & -0.060 & -0.340 & 0.010 & 2.190 & 2.90 & -0.170 \\
\hline Sexual behavior risk & 0.000 & 0.000 & 0.000 & 0.000 & 0.000 & 0.120 & 0.00 & 0.000 \\
\hline No & $0(0.406)$ & $0(0.255)$ & $0(0.599)$ & $0(0.876)$ & $0(0.612)$ & $0.06(0.825)$ & $0.00(0.977)$ & $0(0.838)$ \\
\hline Yes & $0(0.406)$ & $0(0.255)$ & $0(0.599)$ & $0(0.876)$ & $0(0.612)$ & $0.06(0.825)$ & $0.00(0.977)$ & $0(0.838)$ \\
\hline Premarital sex & -0.040 & -0.360 & 0.040 & -0.240 & -0.020 & 1.440 & 2.85 & -1.320 \\
\hline
\end{tabular}


Table 3 Contribution of HIV/AIDS risk factors to gender inequalities in HIV/AIDS prevalence by country; decomposition analysis using latest available DHS, 2003 to 2011 (Continued)

\begin{tabular}{|c|c|c|c|c|c|c|c|c|}
\hline No & $-0.02(0.022)$ & $-0.18(0.007)$ & $0.02(0.543)$ & $-0.12(0.29)$ & $-0.01(0.606)$ & $0.72(0.821)$ & $1.43(0.943)$ & $-0.66(0.000)$ \\
\hline Yes & $-0.02(0.022)$ & $-0.18(0.007)$ & $0.02(0.543)$ & $-0.12(0.29)$ & $-0.01(0.606)$ & $0.72(0.821)$ & $1.42(0.943)$ & $-0.66(0.000)$ \\
\hline Multiple sex partners & 0.020 & -0.360 & -0.020 & 0.000 & -0.020 & 0.200 & -0.06 & -0.200 \\
\hline No & $0.01(0.669)$ & $-0.18(0.003)$ & $-0.01(0.87)$ & $0(0.88)$ & $-0.01(0.607)$ & $0.1(0.825)$ & $0.03(0.942)$ & $-0.1(0.301)$ \\
\hline Yes & $0.01(0.669)$ & $-0.18(0.003)$ & $-0.01(0.87)$ & $0(0.88)$ & $-0.01(0.607)$ & $0.1(0.825)$ & $0.03(0.942)$ & $-0.1(0.301)$ \\
\hline Age at first sex & 0.820 & 0.640 & -0.080 & -0.100 & 0.050 & 0.430 & -0.01 & 1.350 \\
\hline Never had sex & $0.62(0.000)$ & $0.13(0.118)$ & $-0.03(0.336)$ & $0.08(0.317)$ & $0.03(0.629)$ & $0.4(0.829)$ & $-0.05(0.943)$ & $0.73(0.000)$ \\
\hline$<16$ years & $0.23(0.000)$ & $0.27(0.002)$ & $-0.01(0.633)$ & $-0.02(0.785)$ & $0.03(0.678)$ & $0.18(0.83)$ & $-0.08(0.943)$ & $0.27(0.000)$ \\
\hline $16-17$ & $0.19(0.000)$ & $0.07(0.089)$ & $-0.03(0.433)$ & $0.03(0.48)$ & $0(0.704)$ & $-0.16(0.83)$ & $-0.22(0.944)$ & $0.31(0.003)$ \\
\hline 18-19 & $0.06(0.000)$ & $-0.05(0.028)$ & $0.02(0.293)$ & $-0.07(0.308)$ & $0(0.628)$ & $-0.08(0.83)$ & $0.02(0.943)$ & $0.07(0.017)$ \\
\hline $20+$ & $-0.28(0.000)$ & $0.22(0.058)$ & $-0.03(0.589)$ & $-0.12(0.285)$ & $-0.01(0.776)$ & $0.09(0.83)$ & $0.32(0.943)$ & $-0.03(0.823)$ \\
\hline HIV/AIDS awareness & 0.020 & -0.020 & 0.050 & 0.050 & -0.020 & -0.450 & 0.08 & 0.270 \\
\hline Low & $0.01(0.36)$ & $-0.01(0.004)$ & $0.02(0.736)$ & $0.03(0.462)$ & $-0.01(0.642)$ & $-0.15(0.83)$ & $0.04(0.942)$ & $0.12(0.029)$ \\
\hline Average & $0(0.178)$ & $0(0.809)$ & $0(0.85)$ & $0(0.917)$ & $0(0.715)$ & $-0.02(0.829)$ & $0.00(0.951)$ & $-0.02(0.564)$ \\
\hline High & $0.01(0.095)$ & $-0.01(0.002)$ & $0.03(0.621)$ & $0.02(0.475)$ & $-0.01(0.624)$ & $-0.28(0.828)$ & $0.04(0.943)$ & $0.17(0.021)$ \\
\hline The response effect ${ }^{\prime}$ & $0.09(0.811)$ & $0.34(0.476)$ & $2.39(0.000)$ & $2.6(0.013)$ & $0.76(0.039)$ & $1.06(0.028)$ & $3.27(0.002)$ & $4.16(0.000)$ \\
\hline $\begin{array}{l}\text { Socio-economic and } \\
\text { demographic characteristics }\end{array}$ & 0.020 & -3.510 & -0.360 & 5.470 & 0.090 & 0.390 & -0.10 & 3.000 \\
\hline Residence & 0.000 & -0.100 & -0.120 & 0.110 & -0.120 & 0.060 & -0.20 & -0.180 \\
\hline Urban & $0(0.978)$ & $0.03(0.498)$ & $-0.28(0.432)$ & $-0.84(0.086)$ & $0.04(0.433)$ & $-0.02(0.708)$ & $0.63(0.077)$ & $0.13(0.599)$ \\
\hline Rural & $0(0.978)$ & $-0.13(0.498)$ & $0.16(0.432)$ & $0.95(0.086)$ & $-0.16(0.433)$ & $0.08(0.708)$ & $-0.83(0.077)$ & $-0.31(0.599)$ \\
\hline Sex of household head & 0.010 & -0.060 & -0.070 & -0.440 & -0.360 & -0.120 & -0.65 & -0.080 \\
\hline Male & $0.01(0.815)$ & $-0.08(0.554)$ & $-0.08(0.909)$ & $-0.51(0.553)$ & $-0.42(0.071)$ & $-0.15(0.599)$ & $-0.75(0.380)$ & $-0.14(0.746)$ \\
\hline Female & $0(0.815)$ & $0.02(0.554)$ & $0.01(0.909)$ & $0.07(0.553)$ & $0.06(0.071)$ & $0.03(0.599)$ & $0.09(0.380)$ & $0.06(0.746)$ \\
\hline Age group & 0.000 & 0.010 & 0.320 & 0.100 & 0.010 & 0.080 & 0.31 & 0.800 \\
\hline $15-19$ & $0(0.871)$ & $0.12(0.417)$ & $-0.14(0.655)$ & $0.17(0.703)$ & $0.08(0.483)$ & $0.2(0.303)$ & $-0.07(0.877)$ & $-0.49(0.377)$ \\
\hline $20-29$ & $-0.01(0.813)$ & $0.25(0.355)$ & $0.39(0.159)$ & $0.02(0.963)$ & $0.05(0.619)$ & $0.21(0.249)$ & $0.88(0.021)$ & $1.72(0.000)$ \\
\hline $30-39$ & $0(0.821)$ & $-0.1(0.372)$ & $0.34(0.198)$ & $0.13(0.714)$ & $-0.03(0.6)$ & $-0.04(0.61)$ & $0.27(0.400)$ & $0.32(0.238)$ \\
\hline $40+$ & $0.01(0.814)$ & $-0.26(0.342)$ & $-0.27(0.045)$ & $-0.22(0.272)$ & $-0.09(0.214)$ & $-0.29(0.017)$ & $-0.77(0.009)$ & $-0.75(0.000)$ \\
\hline Education level & 0.000 & -0.050 & -0.380 & -0.220 & -0.040 & 0.000 & -0.31 & 1.170 \\
\hline None & $0(0.814)$ & $0.01(0.533)$ & $0.01(0.613)$ & $0.27(0.454)$ & $-0.1(0.29)$ & $-0.03(0.55)$ & $0.03(0.717)$ & $-0.02(0.424)$ \\
\hline Primary & $0(0.816)$ & $0.04(0.705)$ & $-0.05(0.759)$ & $0.28(0.293)$ & $0.02(0.823)$ & $-0.03(0.884)$ & $0.24(0.620)$ & $0.29(0.456)$ \\
\hline Secondary and above & $0(0.899)$ & $-0.1(0.453)$ & $-0.34(0.581)$ & $-0.77(0.119)$ & $0.04(0.344)$ & $0.06(0.519)$ & $-0.58(0.318)$ & $0.9(0.46)$ \\
\hline Standard of living & 0.000 & 0.020 & 0.460 & 0.510 & 0.010 & -0.080 & 0.130 & 0.060 \\
\hline 1st Quintile & $0(0.985)$ & $0.01(0.828)$ & $-0.15(0.115)$ & $-0.52(0.082)$ & $-0.08(0.483)$ & $-0.15(0.178)$ & $0.36(0.281)$ & $-0.12(0.585)$ \\
\hline 2nd Quintile & $0(0.822)$ & $-0.07(0.4)$ & $-0.09(0.383)$ & $-0.34(0.141)$ & $0.09(0.443)$ & $-0.1(0.247)$ & $-0.06(0.742)$ & $0.04(0.848)$ \\
\hline 3rd Quintile & $0(0.818)$ & $0.03(0.539)$ & 0 (0.974) & $0.16(0.594)$ & $0(0.952)$ & $-0.03(0.743)$ & $-0.17(0.396)$ & $0.27(0.261)$ \\
\hline 4th Quintile & $0.000(0.828)$ & $0.02(0.634)$ & $0.27(0.218)$ & $0.000(0.999)$ & $-0.01(0.766)$ & $0.11(0.165)$ & $-0.01(0.946)$ & $0.14(0.571)$ \\
\hline 5th Quintile & $0.000(0.842)$ & $0.03(0.616)$ & $0.43(0.133)$ & $1.21(0.026)$ & $0.01(0.762)$ & $0.09(0.289)$ & $0.01(0.980)$ & $-0.27(0.277)$ \\
\hline Occupation type & 0.010 & -3.280 & & 4.970 & 0.450 & 0.260 & 0.010 & 0.270 \\
\hline Unemployed & $0(0.849)$ & $-0.61(0.349)$ & - & $1.79(0.035)$ & $0.06(0.183)$ & $-0.04(0.518)$ & $0.04(0.901)$ & $-0.35(0.368)$ \\
\hline Agricultural & $0.01(0.814)$ & $-0.72(0.346)$ & - & $2.11(0.037)$ & $0.44(0.131)$ & $0.31(0.339)$ & $-0.23(0.666)$ & $0.02(0.944)$ \\
\hline Domestic & - & $0.09(0.346)$ & - & - & - & $0.02(0.586)$ & - & $-0.06(0.176)$ \\
\hline Trade & $0(0.818)$ & $-0.26(0.348)$ & - & $0.47(0.052)$ & $-0.03(0.313)$ & $0.08(0.077)$ & $0.24(0.129)$ & $0.14(0.227)$ \\
\hline Manual labor & $0(0.813)$ & $-1.36(0.346)$ & - & 0 (0.999) & $0.03(0.368)$ & $-0.07(0.57)$ & $0.04(0.869)$ & $0.38(0.309)$ \\
\hline
\end{tabular}


Table 3 Contribution of HIV/AIDS risk factors to gender inequalities in HIV/AIDS prevalence by country; decomposition analysis using latest available DHS, 2003 to 2011 (Continued)

\begin{tabular}{|c|c|c|c|c|c|c|c|c|}
\hline Office/service & $0(0.815)$ & $-0.21(0.352)$ & - & $1(0.025)$ & $-0.01(0.253)$ & $-0.04(0.092)$ & $-0.12(0.135)$ & $-0.08(0.228)$ \\
\hline Professional/manager & $0(0.819)$ & $-0.21(0.346)$ & - & $-0.4(0.015)$ & $-0.04(0.126)$ & $0(0.851)$ & $0.04(0.740)$ & $0.22(0.059)$ \\
\hline Marital status & 0.000 & -0.050 & -0.570 & 0.440 & 0.140 & 0.190 & 0.630 & 0.960 \\
\hline Never married & $0(0.825)$ & $0.13(0.465)$ & $0.25(0.473)$ & $0.5(0.532)$ & $0.02(0.868)$ & $0.56(0.095)$ & $2.18(0.002)$ & $3.79(0.000)$ \\
\hline Married & $0(0.821)$ & $-0.17(0.424)$ & $-0.92(0.015)$ & $0(0.995)$ & $0.13(0.401)$ & $-0.36(0.162)$ & $-1.39(0.036)$ & $-2.68(0.000)$ \\
\hline $\begin{array}{l}\text { Separated/divorced/ } \\
\text { widowed }\end{array}$ & $0(0.885)$ & $-0.01(0.717)$ & $0.1(0.091)$ & $-0.06(0.456)$ & $-0.01(0.272)$ & $-0.01(0.272)$ & $-0.17(0.009)$ & $-0.15(0.004)$ \\
\hline Sexual behavioral factors ${ }^{f}$ & 0.030 & 0.200 & 0.200 & -0.640 & 0.440 & -0.490 & 1.47 & 1.320 \\
\hline Sexual behavior risk & 0.020 & 0.180 & -0.020 & -0.850 & 0.700 & -0.120 & -0.320 & 1.880 \\
\hline No & $0.02(0.813)$ & $0.21(0.436)$ & $0.08(0.65)$ & $-1.12(0.183)$ & $0.72(0.066)$ & $-0.12(0.858)$ & $-0.36(0.703)$ & $1.98(0.081)$ \\
\hline Yes & $0(0.813)$ & $-0.03(0.436)$ & $-0.1(0.65)$ & $0.27(0.183)$ & $-0.02(0.066)$ & $0(0.858)$ & $0.04(0.703)$ & $-0.1(0.081)$ \\
\hline Premarital sex & 0.000 & 0.070 & 0.290 & -0.130 & 0.010 & 0.080 & -0.010 & 0.000 \\
\hline No & $0.000(0.822)$ & $-0.08(0.419)$ & $-0.06(0.424)$ & $0.06(0.801)$ & $0.01(0.899)$ & $0.27(0.086)$ & $0.01(0.976)$ & $-0.03(0.927)$ \\
\hline Yes & $0.000(0.822)$ & $0.15(0.419)$ & $0.35(0.424)$ & $-0.19(0.801)$ & $0(0.899)$ & $-0.19(0.086)$ & $-0.02(0.976)$ & $0.03(0.927)$ \\
\hline Multiple sex partners & -0.010 & -0.080 & -0.070 & 0.020 & -0.440 & 0.080 & 0.600 & 0.170 \\
\hline No & $-0.01(0.81)$ & $-0.12(0.53)$ & $0.46(0.022)$ & $0.62(0.136)$ & $-0.46(0.116)$ & $0.09(0.756)$ & $0.96(0.104)$ & $0.24(0.69)$ \\
\hline Yes & $0(0.81)$ & $0.04(0.53)$ & $-0.53(0.022)$ & $-0.6(0.136)$ & $0.02(0.116)$ & $-0.01(0.756)$ & $-0.36(0.104)$ & $-0.07(0.69)$ \\
\hline Age at first sex & 0.020 & 0.030 & 0.000 & 0.320 & 0.170 & -0.530 & 1.200 & -0.730 \\
\hline Never had sex & $0.09(0.814)$ & $-0.14(0.412)$ & $-0.03(0.854)$ & $-0.57(0.327)$ & $0.16(0.307)$ & $-0.87(0.068)$ & $-1.17(0.026)$ & $-2.91(0.001)$ \\
\hline$<16$ years & $-0.01(0.814)$ & $0.07(0.387)$ & $0.31(0.399)$ & $-0.15(0.704)$ & $-0.02(0.251)$ & $0.15(0.101)$ & $0.76(0.117)$ & $0.28(0.029)$ \\
\hline $16-17$ & $-0.01(0.815)$ & $0.05(0.479)$ & $-0.31(0.138)$ & $0.17(0.592)$ & $-0.04(0.142)$ & $0.07(0.227)$ & $0.58(0.073)$ & $0.35(0.053)$ \\
\hline $18-19$ & $-0.02(0.814)$ & $0.09(0.404)$ & $-0.14(0.333)$ & $0.67(0.119)$ & $0.03(0.565)$ & $0.14(0.111)$ & $0.35(0.323)$ & $0.53(0.03)$ \\
\hline $20+$ & $-0.03(0.815)$ & $-0.04(0.586)$ & $0.17(0.115)$ & $0.2(0.512)$ & $0.04(0.743)$ & $-0.02(0.863)$ & $0.68(0.060)$ & $1.02(0.014)$ \\
\hline HIV/AIDS awareness & 0.010 & -0.030 & 0.070 & 0.570 & 0.010 & -0.020 & 0.130 & 0.120 \\
\hline Low & $0.000(0.815)$ & $0.000(0.961)$ & $-0.07(0.556)$ & $-0.45(0.048)$ & $-0.05(0.408)$ & $-0.07(0.551)$ & $0.24(0.429)$ & $-0.19(0.445)$ \\
\hline Average & $0(0.819)$ & $-0.07(0.449)$ & $0.02(0.923)$ & $1.34(0.009)$ & $0.09(0.229)$ & $-0.01(0.893)$ & $0.06(0.816)$ & $0(0.997)$ \\
\hline High & $0.01(0.814)$ & $0.04(0.466)$ & $0.12(0.58)$ & $-0.32(0.388)$ & $-0.03(0.716)$ & $0.06(0.437)$ & $-0.18(0.314)$ & $0.31(0.329)$ \\
\hline Constant & $0.04(0.812)$ & $3.68(0.357)$ & $2.48(0.018)$ & $-2.78(0.309)$ & $0.21(0.541)$ & $1.19(0.183)$ & $1.77(0.242)$ & $-0.3(0.885)$ \\
\hline
\end{tabular}

$P$-values are reported in parenthesis; They are testing variables' contribution to gender inequality in HIV/AIDS in each country ${ }^{\mathrm{a} D o m e s t i c}$ category not collected 'Occupation type variable not collected ${ }^{\mathrm{C}}$ Unemployed category not collected ${ }^{\mathrm{d}}$ Trade and domestic category not collected ${ }^{\mathrm{e}}$ The overall absolute contribution of a given variable is equal to the sum of absolute contribution of its categories; the absolute contribution of socio-demographic characteristics, sexual behaviours, and awareness of HIV/AIDS is determined by summing the absolute contributions of all variables included in each category. The absolute contribution of a given variable to a given component was calculated as follow: the absolute difference of HIV/AIDS prevalence between women and men explained by this component

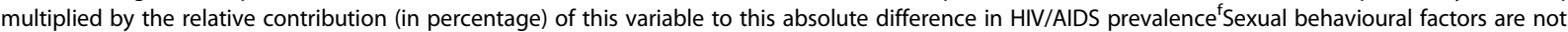
highly correlated ${ }^{9}$ Several values of dichotomous variables for the "difference due to distribution" component became identical after rounding the estimated values ${ }^{\mathrm{h}}$ Represent the contribution to gender inequalities in HIV/AIDS prevalence due to gender differences in the distributions of observable HIV/AIDS risk factors between women and men'Reflect the contribution to gender inequalities in HIV/AIDS due to gender differences in the effects of measured HIV/AIDS risk factors, as well as unmeasured factors not included in the model 
Table 3 Contribution of HIV/AIDS risk factors to gender inequalities in HIV/AIDS prevalence by country; decomposition analysis using latest available DHS, 2003 to 2011 (Continued)

\begin{tabular}{|c|c|c|c|c|c|c|c|}
\hline & $\begin{array}{l}\text { Mozambique } \\
2009\end{array}$ & $\begin{array}{l}\text { Cameroon } \\
2011\end{array}$ & $\begin{array}{l}\text { Guinea }^{a} \\
2005\end{array}$ & $\begin{array}{l}\text { Malawi } \\
2010\end{array}$ & $\begin{array}{l}\text { Swaziland } \\
\text { 2006/07 }\end{array}$ & $\begin{array}{l}\text { Liberia } \\
2007\end{array}$ & $\begin{array}{l}\text { D.R. Congo } \\
2007\end{array}$ \\
\hline & \multicolumn{7}{|c|}{ Absolute contribution ${ }^{e}$ to gender inequalities in HIV/AIDS prevalence ( $p$-value) } \\
\hline $\begin{array}{l}\text { Gender Difference in } \\
\text { HIV/AIDS Prevalence (\%) }\end{array}$ & 3.63 & 2.69 & 0.79 & 4.5 & 11.45 & 0.68 & 0.7 \\
\hline The composition effect ${ }^{\text {hg }}$ & $0.67(0.583)$ & $1.19(0.001)$ & $-1.39(0.05)$ & $2.20(0.00)$ & $2.14(0.015)$ & $-0.76(0.314)$ & $-0.07(0.852)$ \\
\hline $\begin{array}{l}\text { Socio-economic and } \\
\text { demographic characteristics }\end{array}$ & 0.660 & 0.720 & 0.040 & 1.920 & 0.440 & 0.030 & 0.110 \\
\hline Residence & -0.360 & 0.020 & -0.400 & -0.120 & -0.040 & 0.000 & -0.040 \\
\hline Urban & $-0.18(0.126)$ & $0.01(0.07)$ & $-0.2(0.026)$ & $-0.06(0.000)$ & $-0.02(0.018)$ & $0(0.799)$ & $-0.02(0.936)$ \\
\hline Rural & $-0.18(0.126)$ & $0.01(0.07)$ & $-0.2(0.026)$ & $-0.06(0.000)$ & $-0.02(0.018)$ & $0(0.799)$ & $-0.02(0.936)$ \\
\hline Sex of household head & 2.020 & 0.340 & 0.140 & 0.740 & -0.160 & -0.020 & -0.020 \\
\hline Male & $1.01(0.122)$ & $0.17(0.007)$ & $0.07(0.065)$ & $0.37(0.004)$ & $-0.08(0.115)$ & $-0.01(0.474)$ & $-0.01(0.931)$ \\
\hline Female & $1.01(0.122)$ & $0.17(0.007)$ & $0.07(0.065)$ & $0.37(0.004)$ & $-0.08(0.115)$ & $-0.01(0.474)$ & $-0.01(0.931)$ \\
\hline Age group & 0.370 & -0.010 & -0.080 & -0.180 & 0.470 & -0.010 & 0.140 \\
\hline $15-19$ & $0.06(0.316)$ & $-0.01(0.054)$ & $0.01(0.138)$ & $0.27(0.006)$ & $0.45(0.002)$ & $0(0.988)$ & $-0.01(0.934)$ \\
\hline $20-29$ & $0.2(0.119)$ & $0(0.91)$ & $-0.04(0.506)$ & $-0.28(0.006)$ & $0.01(0.001)$ & $0(0.566)$ & $0(0.946)$ \\
\hline $30-39$ & $0.01(0.215)$ & $0.05(0.009)$ & $0.14(0.089)$ & $0.04(0.000)$ & $0.13(0.002)$ & $-0.01(0.73)$ & $-0.01(0.934)$ \\
\hline $40+$ & $0.1(0.106)$ & $-0.05(0.515)$ & $-0.19(0.202)$ & $-0.21(0.006)$ & $-0.12(0.008)$ & $0(0.9)$ & $0.16(0.935)$ \\
\hline Education level & -1.050 & -0.210 & -0.980 & -0.360 & -0.030 & 0.070 & -0.090 \\
\hline None & $-0.7(0.308)$ & $-0.18(0.148)$ & $-0.58(0.071)$ & $-0.12(0.333)$ & $0.01(0.103)$ & $0.04(0.577)$ & $0.2(0.935)$ \\
\hline Primary & $-0.17(0.382)$ & $0(0.054)$ & $-0.02(0.648)$ & $-0.01(0.352)$ & $-0.01(0.123)$ & $0(0.829)$ & $-0.17(0.934)$ \\
\hline Secondary and above & $-0.18(0.548)$ & $-0.03(0.702)$ & $-0.38(0.163)$ & $-0.23(0.111)$ & $-0.03(0.006)$ & $0.03(0.651)$ & $-0.12(0.932)$ \\
\hline Standard of living & -0.060 & -0.090 & 0.220 & -0.070 & 0.010 & -0.030 & 0.020 \\
\hline 1st Quintile & $-0.14(0.144)$ & $-0.04(0.003)$ & $0.04(0.119)$ & $-0.06(0.253)$ & $0.01(0.354)$ & $-0.02(0.571)$ & $0.02(0.934)$ \\
\hline 2nd Quintile & $0(0.368)$ & $-0.01(0.043)$ & $0.01(0.647)$ & $0(0.612)$ & $0(0.412)$ & $0(0.901)$ & $-0.02(0.936)$ \\
\hline 3rd Quintile & $0.05(0.121)$ & $0.01(0.045)$ & $0(0.677)$ & $0.01(0.69)$ & $0(0.687)$ & $0(0.88)$ & $0.01(0.934)$ \\
\hline 4th Quintile & $0(0.157)$ & $-0.04(0.02)$ & $0.01(0.371)$ & $-0.01(0.104)$ & $0(0.356)$ & $-0.01(0.541)$ & $0(0.997)$ \\
\hline 5th Quintile & $0.03(0.701)$ & $-0.01(0.051)$ & $0.16(0.062)$ & $-0.01(0.657)$ & $0(0.345)$ & $0(0.886)$ & $0.01(0.936)$ \\
\hline Occupation type & -2.200 & 0.110 & 0.360 & -0.160 & -0.070 & 0.150 & 0.360 \\
\hline Unemployed & - & $-0.05(0.781)$ & $-0.37(0.019)$ & $-0.45(0.124)$ & $-0.05(0.344)$ & -0.07 (0.619) & $0.02(0.936)$ \\
\hline Agricultural & $-1.11(0.213)$ & $-0.01(0.907)$ & $0.02(0.012)$ & $0.24(0.001)$ & $-0.09(0.077)$ & $0.16(0.45)$ & $0.15(0.935)$ \\
\hline Domestic & $0.02(0.321)$ & $0.01(0.634)$ & - & $0.02(0.039)$ & - & $0(0.534)$ & - \\
\hline Trade & $-0.03(0.571)$ & $0.12(0.198)$ & $1.57(0.006)$ & $0.03(0.763)$ & $0.09(0.039)$ & $-0.03(0.585)$ & - \\
\hline Manual labor & $-1.26(0.051)$ & $0(0.987)$ & $-0.78(0.015)$ & $0.1(0.709)$ & $-0.12(0.192)$ & $0.08(0.51)$ & $-0.03(0.925)$ \\
\hline Office/service & $0.28(0.162)$ & $-0.04(0.104)$ & $0.34(0.008)$ & $-0.18(0.006)$ & $0.01(0.808)$ & $0.01(0.703)$ & $0.01(0.955)$ \\
\hline Professional/manager & $-0.1(0.567)$ & $0.08(0.414)$ & $-0.42(0.023)$ & $0.08(0.212)$ & $0.09(0.022)$ & $0(0.974)$ & $0.21(0.935)$ \\
\hline Marital status & 1.940 & 0.560 & 0.780 & 2.070 & 0.260 & -0.130 & -0.260 \\
\hline Never married & $0.75(0.219)$ & $0.54(0.002)$ & $0.65(0.163)$ & $1.36(0.000)$ & $0.33(0.011)$ & $-0.09(0.471)$ & $-0.21(0.934)$ \\
\hline Married & $0.04(0.423)$ & $-0.16(0.131)$ & $0.11(0.679)$ & $-0.07(0.643)$ & $-0.23(0.008)$ & $-0.02(0.585)$ & $0.02(0.936)$ \\
\hline $\begin{array}{l}\text { Separated/divorced/ } \\
\text { widowed }\end{array}$ & $1.15(0.135)$ & $0.18(0.000)$ & $0.02(0.061)$ & $0.78(0.000)$ & $0.16(0.002)$ & $-0.02(0.461)$ & $-0.07(0.934)$ \\
\hline Sexual behavior factors ${ }^{f}$ & -0.010 & 0.490 & -1.240 & 0.120 & 1.700 & -0.770 & -0.140 \\
\hline Sexual behavior risk & 0.100 & 0.000 & -0.400 & 0.020 & -0.040 & -0.020 & 0.120 \\
\hline No & $0.05(0.448)$ & $0(0.001)$ & $-0.2(0.192)$ & $0.01(0.93)$ & $-0.02(0.163)$ & $-0.01(0.487)$ & $0.06(0.933)$ \\
\hline Yes & $0.05(0.448)$ & $0(0.001)$ & $-0.2(0.192)$ & $0.01(0.93)$ & $-0.02(0.163)$ & $-0.01(0.487)$ & $0.06(0.933)$ \\
\hline Premarital sex & -0.040 & -0.240 & -1.320 & -0.460 & 0.160 & 0.040 & 0.240 \\
\hline
\end{tabular}


Table 3 Contribution of HIV/AIDS risk factors to gender inequalities in HIV/AIDS prevalence by country; decomposition analysis using latest available DHS, 2003 to 2011 (Continued)

\begin{tabular}{|c|c|c|c|c|c|c|c|}
\hline No & $-0.02(0.948)$ & $-0.12(0.345)$ & $-0.66(0.042)$ & $-0.23(0.345)$ & $0.08(0.01)$ & $0.02(0.591)$ & $0.12(0.936)$ \\
\hline Yes & $-0.02(0.948)$ & $-0.12(0.345)$ & $-0.66(0.042)$ & $-0.23(0.345)$ & $0.08(0.01)$ & $0.02(0.591)$ & $0.12(0.936)$ \\
\hline Multiple sex partners & -0.440 & 0.200 & -0.160 & -0.280 & -0.080 & 0.100 & -0.540 \\
\hline No & $-0.22(0.437)$ & $0.1(0.385)$ & $-0.08(0.743)$ & $-0.14(0.403)$ & $-0.04(0.01)$ & $0.05(0.534)$ & $-0.27(0.933)$ \\
\hline Yes & $-0.22(0.437)$ & $0.1(0.385)$ & $-0.08(0.743)$ & $-0.14(0.403)$ & $-0.04(0.01)$ & $0.05(0.534)$ & $-0.27(0.933)$ \\
\hline Age at first sex & 0.370 & 0.530 & 0.640 & 0.840 & 1.660 & -0.890 & 0.040 \\
\hline Never had sex & $0.28(0.136)$ & $0.17(0.052)$ & $0.12(0.426)$ & $0.09(0.069)$ & $1.24(0.004)$ & $-0.74(0.398)$ & $0.02(0.935)$ \\
\hline$<16$ years & $0.5(0.16)$ & $0.36(0.001)$ & $0.46(0.341)$ & $0.07(0.002)$ & $0.23(0.015)$ & $-0.56(0.395)$ & $0.06(0.934)$ \\
\hline $16-17$ & $0.09(0.128)$ & $0.07(0.097)$ & $0.06(0.581)$ & $0.41(0.038)$ & $0.27(0.009)$ & $-0.14(0.393)$ & $0.03(0.936)$ \\
\hline $18-19$ & $-0.34(0.142)$ & $0(0.945)$ & $-0.14(0.294)$ & $0.03(0.307)$ & $0.07(0.015)$ & $0.36(0.403)$ & $0(0.944)$ \\
\hline $20+$ & $-0.16(0.521)$ & $-0.07(0.665)$ & $0.14(0.79)$ & $0.24(0.317)$ & $-0.15(0.023)$ & $0.19(0.413)$ & $-0.07(0.934)$ \\
\hline HIV/AIDS awareness & 0.000 & -0.070 & -0.170 & 0.170 & -0.010 & 0.000 & -0.050 \\
\hline Low & $-0.02(0.474)$ & $-0.09(0.016)$ & $-0.16(0.245)$ & $0.11(0.003)$ & $0(0.809)$ & $0(0.846)$ & $-0.01(0.935)$ \\
\hline Average & $0(0.241)$ & $0.02(0.469)$ & $0(0.263)$ & $0.03(0.06)$ & $0(0.937)$ & $0(0.578)$ & $-0.03(0.934)$ \\
\hline High & $0.02(0.423)$ & $0(0.001)$ & $-0.01(0.906)$ & $0.03(0.077)$ & $-0.01(0.745)$ & $0(0.591)$ & $-0.01(0.934)$ \\
\hline The response effect' & $2.96(0.051)$ & $1.5(0.002)$ & $2.18(0.006)$ & $2.3(0.006)$ & $9.31(0.000)$ & $1.44(0.094)$ & $0.77(0.106)$ \\
\hline $\begin{array}{l}\text { Socio-economic and } \\
\text { demographic characteristics }\end{array}$ & 0.350 & -0.930 & -7.810 & -0.020 & 3.620 & 6.220 & -0.040 \\
\hline Residence & 0.260 & 0.010 & -0.280 & 0.030 & -0.060 & -0.170 & -0.020 \\
\hline Urban & $-0.22(0.437)$ & $0.06(0.702)$ & $0.42(0.103)$ & $-0.01(0.909)$ & $0.04(0.825)$ & $0.34(0.453)$ & $0.06(0.646)$ \\
\hline Rural & $0.48(0.437)$ & $-0.05(0.702)$ & $-0.7(0.103)$ & $0.04(0.909)$ & $-0.1(0.825)$ & $-0.51(0.453)$ & $-0.08(0.646)$ \\
\hline Sex of household head & -1.140 & -0.320 & 0.450 & -0.320 & 0.070 & 1.070 & 0.000 \\
\hline Male & $-1.28(0.183)$ & $-0.38(0.19)$ & $0.5(0.254)$ & $-0.38(0.464)$ & $0.16(0.697)$ & $1.41(0.374)$ & $0(0.984)$ \\
\hline Female & $0.14(0.183)$ & $0.06(0.19)$ & $-0.05(0.254)$ & $0.06(0.464)$ & $-0.09(0.697)$ & $-0.34(0.374)$ & $0(0.984)$ \\
\hline Age group & -0.950 & 0.000 & -0.040 & 0.180 & 2.830 & 0.170 & 0.090 \\
\hline $15-19$ & $0.64(0.117)$ & $0.44(0.015)$ & $-0.23(0.371)$ & $0.67(0.046)$ & $3.59(0.000)$ & $-0.63(0.418)$ & $-0.29(0.063)$ \\
\hline $20-29$ & $1.02(0.092)$ & $0.06(0.694)$ & $-0.01(0.952)$ & $0.1(0.679)$ & $1.32(0.008)$ & $0.04(0.91)$ & $0.11(0.193)$ \\
\hline $30-39$ & $-1.02(0.031)$ & $-0.22(0.018)$ & $0.35(0.088)$ & $-0.15(0.348)$ & $-0.97(0.000)$ & $0.37(0.469)$ & $0.01(0.904)$ \\
\hline $40+$ & $-1.59(0.008)$ & $-0.28(0.016)$ & $-0.15(0.557)$ & $-0.44(0.004)$ & $-1.11(0.000)$ & $0.39(0.417)$ & $0.26(0.102)$ \\
\hline Education level & 0.040 & -0.390 & -0.480 & 0.010 & -0.690 & 0.130 & 0.180 \\
\hline None & $-0.25(0.346)$ & $0.08(0.229)$ & $-0.87(0.034)$ & $-0.04(0.449)$ & $0.08(0.348)$ & $-0.12(0.574)$ & $-0.05(0.187)$ \\
\hline Primary & $-0.16(0.855)$ & $0(0.974)$ & $0.16(0.227)$ & $-0.26(0.419)$ & $0.26(0.365)$ & $0.19(0.58)$ & $0.2(0.145)$ \\
\hline Secondary and above & $0.45(0.278)$ & $-0.47(0.084)$ & $0.23(0.262)$ & $0.31(0.203)$ & $-1.03(0.041)$ & $0.06(0.886)$ & $0.03(0.849)$ \\
\hline Standard of living & -0.310 & 0.000 & -0.140 & 0.020 & -0.150 & 0.130 & -0.360 \\
\hline 1st Quintile & $-0.27(0.608)$ & $-0.03(0.789)$ & $0.16(0.37)$ & $0.17(0.252)$ & $-0.41(0.091)$ & $-0.16(0.673)$ & $-0.62(0.086)$ \\
\hline 2nd Quintile & $-0.69(0.098)$ & $-0.08(0.331)$ & $0.01(0.941)$ & $-0.02(0.85)$ & $-0.12(0.511)$ & $-0.04(0.864)$ & $-0.62(0.065)$ \\
\hline 3rd Quintile & $0.47(0.135)$ & $0.13(0.094)$ & $0.13(0.388)$ & $-0.06(0.665)$ & $-0.01(0.98)$ & $-0.02(0.899)$ & $-0.68(0.057)$ \\
\hline 4th Quintile & $0.18(0.411)$ & $0.07(0.524)$ & $-0.02(0.839)$ & $-0.09(0.427)$ & $-0.28(0.223)$ & $0.44(0.425)$ & $0.72(0.05)$ \\
\hline 5th Quintile & $0(0.998)$ & $-0.09(0.39)$ & $-0.42(0.107)$ & $0.02(0.926)$ & $0.67(0.011)$ & $-0.09(0.635)$ & $0.84(0.055)$ \\
\hline Occupation type & 2.470 & 0.010 & -0.430 & -0.020 & 0.000 & 5.260 & 0.150 \\
\hline Unemployed & - & $-0.08(0.571)$ & $0.27(0.319)$ & $-0.07(0.633)$ & $-0.04(0.937)$ & $1.23(0.311)$ & $-0.04(0.71)$ \\
\hline Agricultural & $1.59(0.148)$ & $-0.02(0.898)$ & $-0.29(0.403)$ & $-0.21(0.554)$ & $0.07(0.628)$ & $3.16(0.365)$ & $0.19(0.202)$ \\
\hline Domestic & $-0.06(0.368)$ & $-0.05(0.143)$ & - & $0(0.823)$ & - & $-0.05(0.342)$ & - \\
\hline Trade & $0.61(0.061)$ & $0.05(0.427)$ & $-0.19(0.017)$ & $0.23(0.042)$ & $0.07(0.403)$ & $0.24(0.439)$ & - \\
\hline Manual labor & $0.53(0.315)$ & $0.03(0.861)$ & $-0.15(0.204)$ & $0.06(0.76)$ & $0.04(0.872)$ & $0.14(0.527)$ & $0.06(0.47)$ \\
\hline
\end{tabular}


Table 3 Contribution of HIV/AIDS risk factors to gender inequalities in HIV/AIDS prevalence by country; decomposition analysis using latest available DHS, 2003 to 2011 (Continued)

\begin{tabular}{|c|c|c|c|c|c|c|c|}
\hline Office/service & $-0.19(0.294)$ & $0.12(0.029)$ & $0.05(0.003)$ & $0.02(0.736)$ & $0.01(0.966)$ & $0.34(0.38)$ & $-0.06(0.122)$ \\
\hline Professional/manager & $-0.01(0.953)$ & $-0.04(0.435)$ & $-0.12(0.068)$ & $-0.05(0.32)$ & $-0.15(0.308)$ & $0.2(0.43)$ & $0(0.946)$ \\
\hline Marital status & -0.020 & -0.240 & -6.890 & 0.080 & 1.620 & -0.370 & -0.080 \\
\hline Never married & $0.25(0.569)$ & $0.04(0.851)$ & $-2.7(0.004)$ & $0.73(0.175)$ & $2.6(0.001)$ & $1.17(0.389)$ & $-0.01(0.954)$ \\
\hline Married & $-0.2(0.885)$ & $-0.3(0.168)$ & $-4.76(0.001)$ & $-0.62(0.216)$ & $-0.96(0.001)$ & $-1.51(0.395)$ & $-0.08(0.614)$ \\
\hline $\begin{array}{l}\text { Separated/divorced/ } \\
\text { widowed }\end{array}$ & $-0.07(0.46)$ & $0.02(0.318)$ & $0.57(0.001)$ & $-0.03(0.33)$ & $-0.02(0.631)$ & $-0.03(0.679)$ & $0.01(0.674)$ \\
\hline Sexual behavioral factors ${ }^{f}$ & 0.960 & -0.650 & 0.330 & -0.120 & 0.490 & -2.550 & -0.190 \\
\hline Sexual behavior risk & -0.240 & -0.520 & -0.120 & -0.990 & 1.160 & 0.000 & -0.250 \\
\hline No & $-0.3(0.743)$ & $-0.64(0.019)$ & $-0.15(0.772)$ & $-1.13(0.1)$ & $1.4(0.015)$ & $0(0.99)$ & $-0.36(0.18)$ \\
\hline Yes & $0.06(0.743)$ & $0.12(0.019)$ & $0.03(0.772)$ & $0.14(0.1)$ & $-0.24(0.015)$ & $0(0.99)$ & $0.11(0.18)$ \\
\hline Premarital sex & -0.590 & -0.210 & 0.090 & -0.010 & -0.010 & -0.170 & -0.080 \\
\hline No & $0.58(0.112)$ & $0.2(0.08)$ & $-0.07(0.615)$ & $0.000(0.979)$ & $0.01(0.971)$ & $0.06(0.712)$ & $0.05(0.412)$ \\
\hline Yes & $-1.17(0.112)$ & $-0.41(0.08)$ & $0.16(0.615)$ & $-0.01(0.979)$ & $-0.02(0.971)$ & $-0.23(0.712)$ & $-0.13(0.412)$ \\
\hline Multiple sex partners & -0.040 & 0.050 & 0.140 & 0.510 & 0.130 & 0.160 & 0.200 \\
\hline No & $-0.07(0.935)$ & $0.17(0.308)$ & $0.3(0.431)$ & $0.68(0.15)$ & $0.27(0.551)$ & $0.73(0.422)$ & $0.35(0.133)$ \\
\hline Yes & $0.03(0.935)$ & $-0.12(0.308)$ & $-0.16(0.431)$ & $-0.17(0.15)$ & $-0.14(0.551)$ & $-0.57(0.422)$ & $-0.15(0.133)$ \\
\hline Age at first sex & 1.830 & 0.030 & 0.220 & 0.370 & -0.790 & -2.540 & -0.060 \\
\hline Never had sex & $-0.31(0.371)$ & $-0.21(0.45)$ & $-0.41(0.161)$ & $-0.58(0.169)$ & $-1.49(0.203)$ & $2.39(0.347)$ & $0.09(0.398)$ \\
\hline$<16$ years & $0.89(0.262)$ & $0.1(0.342)$ & $0.1(0.507)$ & $0.48(0.126)$ & $0.27(0.105)$ & $-0.88(0.347)$ & $-0.03(0.785)$ \\
\hline $16-17$ & $0.64(0.358)$ & $0.19(0.148)$ & $0.16(0.343)$ & $0.14(0.319)$ & $-0.19(0.324)$ & $-1.53(0.344)$ & $-0.1(0.222)$ \\
\hline $18-19$ & $0.34(0.58)$ & $-0.02(0.89)$ & $0.3(0.146)$ & $0.12(0.464)$ & $0.55(0.043)$ & $-1.87(0.351)$ & $0.01(0.81)$ \\
\hline $20+$ & $0.27(0.663)$ & $-0.03(0.778)$ & $0.07(0.807)$ & $0.21(0.361)$ & $0.07(0.814)$ & $-0.65(0.389)$ & $-0.03(0.646)$ \\
\hline HIV/AIDS awareness & 0.130 & -0.010 & 0.040 & 0.030 & -0.040 & -0.030 & 0.090 \\
\hline Low & $0.92(0.07)$ & $-0.34(0.014)$ & $-0.15(0.346)$ & $0.01(0.94)$ & $0.01(0.966)$ & $0.26(0.477)$ & $0.96(0.118)$ \\
\hline Average & $-0.76(0.064)$ & $-0.11(0.347)$ & $0.43(0.085)$ & $0.15(0.375)$ & $-0.1(0.732)$ & $-0.52(0.373)$ & $0.67(0.049)$ \\
\hline High & $-0.03(0.931)$ & $0.44(0.004)$ & $-0.24(0.242)$ & $-0.13(0.351)$ & $0.05(0.794)$ & $0.23(0.509)$ & $-1.54(0.061)$ \\
\hline Constant & $1.52(0.585)$ & $3.09(0.000)$ & $9.61(0.001)$ & $2.4(0.034)$ & $5.22(0.000)$ & $-2.19(0.477)$ & $0.92(0.126)$ \\
\hline
\end{tabular}

prevalence (Cameroon, Guinea, Malawi, and Swaziland), age contributed to gender inequalities in HIV/AIDS prevalence. Results from the $\mathrm{BO}$ decomposition imply that setting the distributions of age to be the same for women and men and equalizing the effect of age on HIV/AIDS prevalence would lead to a $28.8 \%$ decrease in the excess prevalence of HIV/AIDS among women in Swaziland. However, doing so in Guinea and Cameroon would increase these inequalities by $15.2 \%$. The differential effect of age did not contribute to gender inequality in HIV/AIDS in Malawi. In all four of these countries, the constant term, representing the effects of unmeasured factors, made the largest contribution to gender inequalities.

Seven countries were surveyed twice between 2003 and 2012. In six of the seven earlier surveys there was a significant difference in the prevalence of HIV/AIDS between women and men. We examined the sources of gender inequality in HIV/AIDS in these six surveys, fielded between 2004 and 2006 (Additional file 1: Table S2). Similar to our main findings, analyses of these six additional surveys showed that, in most countries, inequalities in HIV/AIDS prevalence between men and women were attributable to the differences in the responses to HIV/ AIDS risk factors (Additional file 1: Table S3). The response effect of unmeasured characteristics made the largest contribution to gender inequalities in HIV/AIDS prevalence in most of these six countries. Additionally, the main sources of gender inequalities in HIV/AIDS within countries changed over time in Cameroon, Ethiopia, Malawi and Zimbabwe.

\section{Discussion}

The global burden of HIV/AIDS varies considerably between countries, with those in southern Africa being most affected by the pandemic [60]. We estimated the absolute difference in HIV/AIDS prevalence comparing women to men in 21 SSA countries and identified 


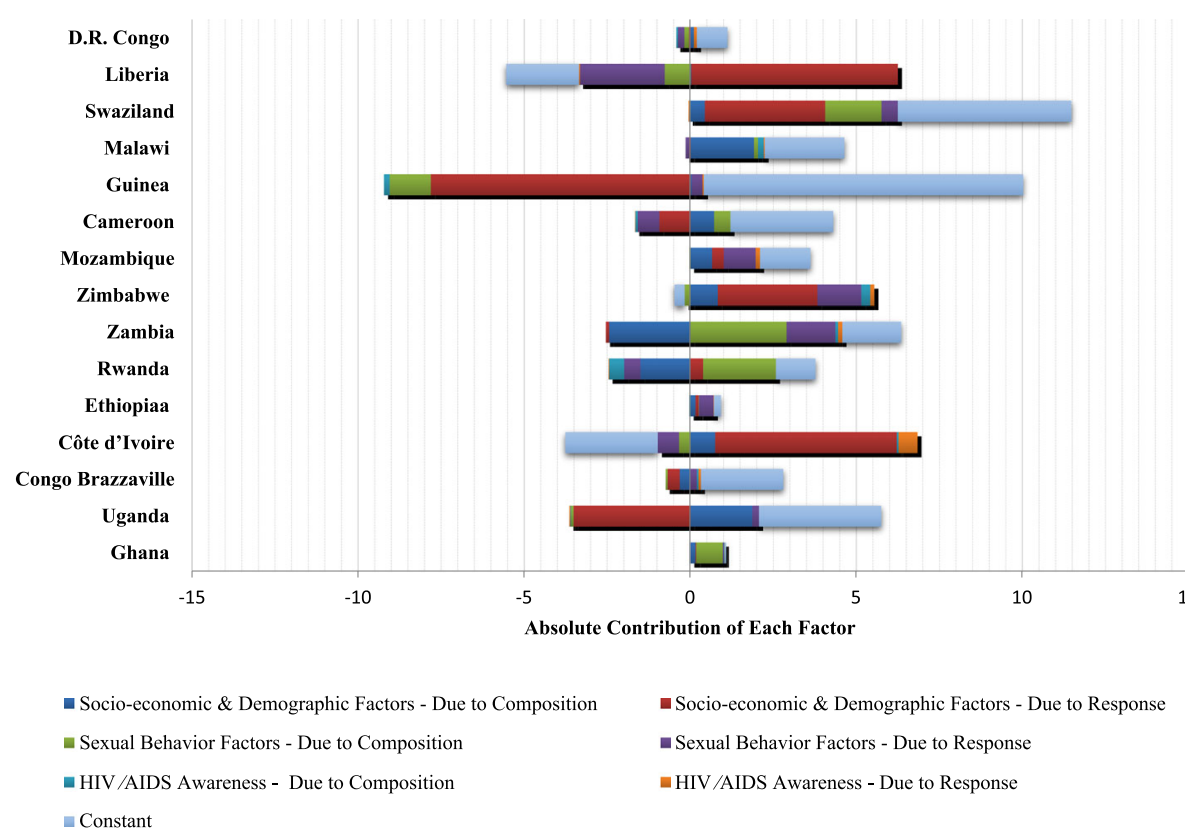

Fig. 3 Contribution of factors to gender inequalities in HIV/AIDS prevalence by country

sources of gender inequalities. Descriptive analyses showed that gender inequalities in HIV/AIDS prevalence were most pronounced in the south-east region of SSA, where socioeconomic inequalities in HIV/AIDS are also greater relative to other SSA countries [61]. In these countries in particular, gender inequalities in HIV/AIDS prevalence were primarily explained by the differential effects of HIV/AIDS risk factors for men and women rather than by the differential distributions of these characteristics. Reducing gender inequalities might be essential to efforts to eliminate HIV/AIDS [62].

There are gender inequalities in the prevalence of HIV/AIDS in SSA across countries. Our analyses showed that women had a higher prevalence of HIV/ AIDS than men in at least three-quarters of the countries surveyed. Furthermore, a comparison of gender inequalities within countries surveyed on two occasions since 2003 suggests that these inequalities are persistent over time. Consistent with earlier work [63], our results showed that gender inequalities in HIV/AIDS prevalence were larger in magnitude in countries with a greater burden of HIV/AIDS, particularly countries situated in south-east SSA, including Malawi, Mozambique, Swaziland, Zambia, and Zimbabwe. A higher probability of HIV transmission [64], as well as greater community viral load (CVL) [65], may contribute to greater prevalence of HIV/AIDS in this region. A systematic review of observational studies by Boily and colleagues [64] suggested that regional differences might be explained by variations in contraceptive practices, differential burden of viral subtypes, and interaction with other infectious diseases, among other factors. Additionally, a recent study by Abu-Raddad and colleagues [65] indicated that viral load is higher in SSA than other regions, and it is highest in southern and east Africa. Community viral load may be a central driver of the HIV epidemic in SSA, where it may reflect, among other factors, the high burden of coinfections such as malaria, tuberculosis and other tropical diseases or the preponderance of HIV-1 subtype C infection. Other work suggests that the level of wealth inequality in SSA region is associated with HIV prevalence [65-67].

Results from our decomposition analysis showed that the sources of gender inequality in HIV/AIDS vary across countries. In most of the countries surveyed, gender inequalities in HIV/AIDS were primarily explained by differences in the effects of risk factors-both measured (i.e. socio-demographic characteristics, sexual behaviours, and HIV/AIDS awareness) and unmeasured in our model-on HIV/AIDS seropositivity for women and men. Gender-related constraints, including women's limited control of resources, may decrease women's ability to protect themselves against diseases [68] and explain our observations. For example, with respect to measured socio-demographic characteristics, the differential effects of occupation contributed to the disproportionate burden of HIV/AIDS among women in SSA. The same occupational classes may be associated with different risks for HIV/AIDS for women compared to men. For example, research indicates that unemployed women generally face poorer job prospects than unemployed men [69]. Further, unemployed women are more economically dependent on their male partners and have fewer 
alternatives to protect themselves against disease transmission [68]. Similarly, marital status has different implications for women and men in many contexts because of cultural restrictions on women's autonomy in the public sphere. These constraints may contribute to gender inequalities in HIV/AIDS by reducing the capacity of unmarried women to engage in equitable relationships and negotiate safe sexual practices (e.g. condom use) with their partners [70, 71], which increases the probability of HIV infection. Other research showed that women who exited an abusive marriage were likely to enter another one with new risks [72], or enter domestic service, which is associated with workplace violence [73].

In a subset of countries, differences in the distributions of sexual behaviors, including age at first sex and premarital sex, between women and men were the main factors contributing to gender inequalities in HIV/AIDS. The age of first sex was lower, on average, for women compared to men, suggesting they were at higher risk of HIV infection due to a longer risk period [74]. Consistent with earlier work [75-78], interventions that delay the age at which women experience intercourse might reduce gender inequalities in HIV/AIDS. Indeed, it has been shown that a longer duration of premarital sex relative to the duration of marriage was associated with an increased odds of HIV infection and other sexually transmitted diseases [79]. In our sample, women were less likely to have premarital sex compared to men (see descriptive analysis in Additional file 1: Table S1). This likely protected women against HIV/AIDS. Moreover, our analysis showed that in countries where composition and response sources of gender inequalities in HIV/AIDS played a significant role (e.g. Cameroon, Guinea, Malawi and Swaziland), the differential effects of unmeasured factors made a large and significant contribution. Unmeasured factors that might contribute to gender inequalities in HIV/AIDS include power processes through couple communication and collaboration [80], lack of female empowerment and limited access to health resources [40], social support [81], migration [82], and lack of an enabling environment for women [83]. Also, several unmeasured biological mechanisms might increase women's risk of contracting HIV [84]. First, research indicates that during sexual intercourse women have a greater mucosal surface area exposed to infectious fluid for longer periods and are more likely to face increased tissue injury [85]. Second, sexually transmitted infectious diseases increase the risk of contracting HIV, particularly for women, because these infections are often asymptomatic and untreated [85-87]. Third, women have a window of vulnerability after ovulation in which the potential for viral infectivity in the female reproductive tract is increased [88]. Further work is required to understand how the differential effects of risk factors contribute to gender inequalities in HIV/AIDS.
Our study is subject to some potential limitations. First, although the DHS/AIS provide nationally representative estimates of HIV prevalence, our results could be biased due to the voluntary nature of the HIV test. Nevertheless, prior studies [24, 89] showed that nonresponse was unlikely to bias national estimates of prevalence from the DHS. Further, it has been shown that non-response is more likely to be random than selective in the DHSs [3]. Second, the cross-sectional nature of data cannot establish temporality between risk factors and outcome status, making it impossible to rule out reverse causality-our findings should therefore be interpreted as associations rather than causal estimates of the impact of intervening on HIV/AIDS risk factors. Third, because we used individual-level data we could not quantify the contribution of structural factors (e.g., CVL, wealth inequality) to gender inequalities in $\mathrm{HIV} /$ AIDS. In brief, this is because structural factors operate at the aggregate-level and cannot be used to decompose individual-level gender inequalities in HIV/AIDS prevalence. We also could not incorporate biological factors in our analyses, as this information was not available in our datasets. Finally, HIV risk factors were self-reported and may be reported with error; for example, individuals might misreport sexual behaviors [90].

Caveats considered, the results of this study have some useful implications for future research and for potential interventions targeting gender inequalities in HIV/AIDS in SSA. First, in the majority of countries it was the differential effects of measured and unmeasured HIV/AIDS risk factors that contributed to gender inequalities. Further research, including sub-national research and mixed methods approaches, is needed to identify these risk factors and elucidate how they interact with gender to exacerbate differences in the burden of HIV/AIDS between women and men. Second, when these processes are better understood, our findings suggest that countryspecific interventions and preventive programs based on the sources of gender inequalities in each context [37, 38] will be needed to mitigate gender inequalities in $\mathrm{HIV} /$ AIDS in SSA. Given the different patterns that we observed in the sources and factors contributing to gender inequalities across countries, general interventions to reduce gender inequalities in HIV/AIDS may not be effective [37]. Prior work [91-97] has proposed a structural approach to HIV prevention which takes into account the main dimensions of women's empowerment; examining whether interventions designed to empower women reduce inequalities in HIV/AIDS is a fruitful area for further research.

\section{Conclusions}

Using the most recent available data we measured gender inequalities in HIV/AIDS prevalence in the SSA 
region and identified sources and factors contributing to these inequalities. We found three unique patterns: i) countries where gender inequality in HIV/AIDS prevalence was due to differences in the distribution of risk factors between men and women, ii) countries where gender inequalities in HIV/AIDS prevalence were due to differences in the effects of risk factors on prevalence of HIV/AIDS for women and men, and iii) countries where the combination of risk factors being distributed differently and having differential effects for men and women explained gender inequalities in HIV/AIDS prevalence. In countries (e.g. Cameroon, Guinea, Malawi, and Swaziland) where unmeasured characteristics substantially contributed to gender inequalities, future work is required to understand the factors underpinning inequalities. Moreover, by adding to extant knowledge concerning the determinants of gender inequalities in HIV/AIDS in SSA, our study can help prioritize interventions to tackle gender inequalities in HIV/AIDS prevalence.

\section{Additional file}

Additional file 1: This document provides three tables titled "Table S1. Sample characteristics by gender and surveys", "Table S2. Results from Blinder-Oaxaca decomposition analysis of gender inequalities in HIV/AIDS prevalence using earlier DHSs conducted between 2004 and 2006, for countries surveyed twice between 2003 and 2012" and "Table S3. Results from Blinder-Oaxaca decomposition analysis of gender inequalities in HIV/AIDS prevalence using earlier DHSs conducted between 2004 and 2006, for countries surveyed twice between 2003 and 2012". (DOCX 110 kb)

\section{Acknowledgments}

The authors gratefully acknowledge MEASURE DHS for their permission to use the datasets.

\section{Funding}

DS and $\mathrm{MH}$ are funded by the Strategic Training Program in Global Health Research, a partnership of the Canadian Institutes of Health Research (CIHR) and the Québec Population Health Research Network (QPHRN). JH and AN acknowledge the support of the Canada Research Chairs Program. DS, MH, $J H, T B$ and AN acknowledge the support of the CIHR Operating Grant: Examining the impact of social policies on health equity $(\mathrm{ROH}-115209)$.

\section{Availability of data and materials}

We used secondary data from Demographic Health Survey (http:// www.dhsprogram.com/). We confirm that all data underlying the findings are fully available without restriction. We received permission to use this data from MEASURE DHS, which is the monitoring body of the DHS.

\section{Authors' contributions}

All authors contributed to the conception and design of the study, DS performed the statistical analysis, all authors interpreted results, DS drafted the manuscript, and $\mathrm{YO}, \mathrm{MH}, \mathrm{JH}, \mathrm{TB}$ and $\mathrm{AN}$ helped with drafting and revisions. All authors read and approved the final version of the manuscript.

\section{Competing interests}

The authors declare that they have no competing interests.

\section{Consent for publication}

Not applicable.

\section{Ethics approval and consent to participate}

We used secondary data from the international Demographic and Health Survey (DHS) program and the AIDS Indicator Survey (AIS). This work does not involve animal experimentation or human subjects and was exempted from committee review.

\section{Author details}

'Département des sciences infirmières, Campus de Saint-Jérôme, Université du Québec en Outaouais, 5, rue Saint-Joseph, bureau J-3226, Saint Jérôme, Québec J7Z 0B7, Canada. ${ }^{2}$ Institut Supérieur des Sciences de la Population (ISSP), Université Ouaga I Pr Joseph Ki-Zerbo, 03 BP 7118 Ouagadougou 03, Ouagadougou, Burkina Faso. ${ }^{3}$ School of Health Administration, Dalhousie University, Halifax, Nova Scotia, Canada. ${ }^{4}$ Fielding School of Public Health, The University of California, Los Angeles (UCLA), Los Angeles, CA, USA. ${ }^{5}$ David Geffen School of Medicine, The University of California, Los Angeles (UCLA), Los Angeles, CA, USA. Institute for Health and Social Policy and Department of Epidemiology, Biostatistics, and Occupational Health, McGill University, Montreal, Québec, Canada.

Received: 2 January 2016 Accepted: 19 October 2016

Published online: 03 November 2016

\section{References}

1. ONUSIDA. Journée mondial SIDA. ONUSIDA; 2011. http://www.unaids.org/ sites/default/files/media_asset/JC2216_WorldAIDSday_report_2011_fr_0.pdf. [Accessed 20 Apr 2014].

2. United Nations. The Millennium Development Goals Report 2011. New York: United Nation; 2011. http://www.un.org/millenniumgoals/pdf/(2011_ E)\%20MDG\%20Report\%202011_Book\%20LR.pdf.

3. Magadi MA. Understanding the gender disparity in HIV infection across countries in sub-Saharan Africa: evidence from the demographic and health surveys. Sociol Health IIIn. 2011;33(4):522-39.

4. Pettifor AE, Rees HV, Kleinschmidt I, Steffenson AE, MacPhail C, Hlongwa-Madikizela L, Vermaak K, Padian NS. Young people's sexual health in South Africa: HIV prevalence and sexual behaviors from a nationally representative household survey. AIDS. 2005;19(14):1525-34.

5. Carael M, Marais H, Polsky J, Mendoza A. Is there a gender Gap in the HIV response? Evaluating national HIV responses from the United Nations General Assembly special session on HIV/AIDS country reports. J Acquir Immune Defic Syndr. 2009;52(Supplement 2):S111-8.

6. Beegle K, Ozler B. Young Women, Rich(er) Men, and the Spread of HIV. Washington: World Bank; 2007.

7. Gillespie S. Poverty, food insecurity, HIV vulnerability, and the impacts of AIDS in Sub-Saharan Africa. IDS Bulletin. 2008;39(5):10-8.

8. Piot P. 'AIDS: Exceptionalism Revisited', lecture presented at London School of Economics and Political Science. London: UNAIDS; 2008.

9. Mojola SA. Fishing in dangerous waters: ecology, gender and economy in HIV risk. Soc Sci Med. 2011:72(2):149-56.

10. Bandali S. Exchange of sex for resources: HIV risk and gender norms in Cabo Delgado, Mozambique. Cult Health Sex. 2011;13(5):575-88.

11. Njue C, Voeten HACM, Remes P. Porn video shows, local brew, and transactional sex: HIV risk among youth in Kisumu, Kenya. BMC Public Health. 2011;11:635.

12. Amaro H. Love, sex, and power: considering women's realities in HIV prevention. Am Psychol. 1995;50(6):437-47.

13. Audet C, Burlison J, Moon T, Sidat M, Vergara A, Vermund S. Sociocultural and epidemiological aspects of HIV/AIDS in Mozambique. BMC Int Health Hum Rights. 2010;10(15):1-20.

14. Bouare O. Modeling contextual determinants of HIV/AIDS prevalence in South Africa to inform policy. Afr J Reprod Health. 2009;13(3):53-70.

15. Gupta GR. How men's power over women fuels the HIV epidemic. BMJ. 2002;324(7331):183-4.

16. Jewkes RK, Dunkle K, Nduna M, Shai N. Intimate partner violence, relationship power inequity, and incidence of HIV infection in young women in South Africa: a cohort study. Lancet. 2010;376(9734):41-8.

17. Magadi MA. The disproportionate high risk of HIV infection among the urban poor in Sub-Saharan Africa. AIDS Behav. 2013;17:1645-54.

18. Zierler S, Krieger N. Reframing women's risk: social inequalities and HIV infection. Annu Rev Public Health. 1997;18(1):401-36.

19. Tsai AC, Subramanian SV. Proximate context of gender-unequal norms and women's HIV risk in sub-Saharan Africa. AIDS. 2012;26(3):381-6. 
20. Shannon K, Leiter K, Phaladze N, Hlanze Z, Tsai AC, Heisler M, lacopino V, Weiser SD. Gender inequity norms are associated with increased maleperpetrated rape and sexual risks for HIV infection in Botswana and Swaziland. PLoS One. 2012;7(1):e28739.

21. de Walque D. Does education affect HIV status? Evidence from five African countries. World Bank Econ Rev. 2009;23(2):209-33.

22. Measure DHS. Demographic and health surveys. Calverton: Measure DHS; 2009.

23. Institut national de la statistique du Cameroun, Macro International Inc : Enquête démographique et de santé Cameroun 2004, Calverton, MD, USA: Macro International Inc; 2005. p. 1-479.

24. Mishra V, Vaessen M, Boerma JT, Arnold F, Way A, Barrere B, Cross A Hong R, Sangha J. HIV testing in national population-based surveys: experience from the demographic and health surveys. Bull World Health Organ. 2006:84(7):537-45.

25. Institut national de la statistique du Cameroun. Enquête par grappes à indicateurs multiples 2006, Yaoundé, YDÉ, Cameroun: Institut national de la statistique du Cameroun; 2006. p. 1-355.

26. Reniers G. Marital strategies for regulating exposure to HIV. Demography. 2008;45(2):417-38

27. Watkins SC. Navigating the AIDS epidemic in rural Malawi. Popul Dev Rev. 2004;30(4):673-705.

28. Joesoef MR, Cheluget B, Marum LH, Wandera C, et al. Differential of HIV prevalence in women and men who attended sexually transmitted disease clinics at HIV sentinel surveillance sites in Kenya, 1990-2001. Int J STD AIDS. 2003;14(3):193.

29. Bandali S. Norms and practices within marriage which shape gender roles, HIV/AIDS risk and risk reduction strategies in Cabo Delgado, Mozambique. AIDS Care. 2011;23(9):1171-6.

30. Test FS, Mehta SD, Handler A, Mutimura E, Bamukunde AM, Cohen M. Gender inequities in sexual risks among youth with HIV in Kigali, Rwanda. Int J STD AIDS. 2012;23(6):394-9.

31. Magadi $M$, Desta M. A multilevel analysis of the determinants and cross-national variations of HIV seropositivity in sub-Saharan Africa: evidence from the DHS. Health Place. 2011;17(5):1067-83.

32. Galvin SR, Cohen MS. The role of sexually transmitted diseases in HIV transmission. Nat Rev Microbiol. 2004;2(1):33-42.

33. Glynn JR, Caraël M, Auvert B, Kahindo M, Chege J, Musonda R, Kaona F, Buvé A, Cities ISGotHoHEiA. Why do young women have a much higher prevalence of HIV than young men? A study in Kisumu, Kenya and Ndola, Zambia. AIDS. 2001;15:S51-60.

34. Quinn TC, Overbaugh J. HIV/AIDS in women: an expanding epidemic. Science. 2005;308(5728):1582-3.

35. Temah CT. What drives HIV/AIDS epidemic in Sub-Saharan Africa? Revue d'économie du développement. 2009;23:41-70.

36. United Nations Development Programme. Human development reports 2011 - sustainability and equity: a better future for All. New York: United Nations Development Programme; 2011.

37. Chandler CL. Creating a different international HIV response for young people. Glob Public Health. 2011;6(Supplement 3):S344-56.

38. UNAIDS. 22nd Meeting of the UNAIDS Programme Coordinating Boar: Gender-sensitivity of AIDS Responses. Chiang Mai: UNAIDS; 2008.

39. Sia D, Onadja Y, Nandi A, Foro A, Brewer T. What lies behind gender inequalities in HIV/AIDS in sub-Saharan African countries: evidence from Kenya, Lesotho and Tanzania. Health Policy Plan. 2013;29(7):938-49.

40. Austin KF, Noble MD. Measuring gender disparity in the HIV pandemic: a cross-national investigation of female empowerment, inequality, and disease in less-developed nations. Sociol Inq. 2014;84(1):102-30.

41. Rutstein SO, Rojas G. Guide to DHS statistics. Calverton: ORC Macro; 2003.

42. Macro International Inc. Sampling manual - DHS-III. Basic documentation No. 6. Calverton, Maryland, USA: ICF International; 1996.

43. Measure DHS. Demographic and Health Survey Sampling and Household Listing Manual. Calverton: ICF International; 2012. http://dhsprogram.com/ pubs/pdf/DHSM4/DHS6_Sampling_Manual_Sept2012_DHSM4.pdf.

44. ICF Macro. Demographic and Health Survey Interviewer's Manual MEASURE DHS Basic Documentation No. 2. Calverton: ICF Macro; 2009.

45. DHS M. Description of the Demographic and Health Surveys Individual Recode Data File. Calverton: Measure DHS; 2008. http://dhsprogram.com/ pubs/pdf/DHSG4/Recode4DHS.pdf.

46. Hancioglu A, Arnold F. Measuring coverage in $\mathrm{MNCH}$ : tracking progress in health for women and children using DHS and MICS household surveys. PLoS Med. 2013;10(5):e1001391.
47. Pullum TW. An assessment of the quality of data on health and nutrition in the DHS surveys, 1993-2003. Calverton: Macro International Inc; 2008.

48. Wirth ME, Wirth E, Delamonica E, Sacks D, Balk A, Minujin A. Monitoring health equity in the MDGs: a practical guide. New York: CIESIN/UNICEF; 2006.

49. Kobiané J-F. Essai de construction d'un profil de pauvreté des ménages à Ouagadougou à partir des caractéristiques de I'habitat. In: Gendreau F, editor. Crises, pauvreté et changements démographiques dans les pays du Sud. Paris: AUPELF-UREF, Éditions ESTEM; 1998. p. 117-31.

50. Kobiané J-F. Habitat et biens d'équipement comme indicateurs de niveau de vie des ménages: bilan méthodologique et application à la relation pauvreté/scolarisation. Études de la Population Africaine. 2005; 19(supplément A):265-83.

51. Sia D, Kobiané J-F, Sondo KB, Fournier P. Les facteurs individuels et du milieu de vie associés à la vaccination complète des enfants en milieu rural au Burkina Faso: une approche multiniveau. Cahiers Santé. 2007;17(4):201-6.

52. Blinder A. Wage discrimination : reduces form and structural estimates. J Hum Resour. 1973;8(4):436-55.

53. Oaxaca R. Male-female wage differentials in urban labor markets. Int Econ Rev. 1973;14(3):693-709.

54. Powers DA, Yoshioka H, Yun M-S. mvdcmp: multivariate decomposition for nonlinear response models. Stata J. 2011;11(4):556-76.

55. Even WE, Macpherson DA. The decline of private-sector unionization and the gender wage gap. J Hum Resour. 1993;28:279-96.

56. Fairlie RW. An extension of the Blinder-Oaxaca decomposition technique to logit and probit models. J Econ Soc Meas. 2005;30:305-16.

57. Nielsen HS. Discrimination and detailed decomposition in a logit model. Econ Lett. 1998;61:115-20.

58. Sinning M, Hahn M, Bauer TK. The Blinder-Oaxaca decomposition for nonlinear regression models. Stata J. 2008;8:480-92.

59. Yun M-S. Decomposing differences in the first moment. Econ Lett. 2004:82:275-80.

60. UNAIDS. Global report: UNAIDS report on the global AIDS epidemic Geneva: 2010 available at: http://www.unaids.org/globalreport/documents/ 20101123 GlobalReport full_en.pdf. [Accessed 22 Oct 2016].

61. Hajizadeh M, Sia D, Heymann S, Nandi A. Socioeconomic inequalities in HIV/AIDS prevalence in sub-Saharan African countries: evidence from the Demographic Health Surveys. Int J Equity Health. 2014;13(1):18.

62. Shisana O, Davids A. Correcting gender inequalities is central to controlling HIV/AIDS. In: Bulletin of the World Health Organization. 82nd ed. 2004 p. 812.

63. ICF International. HIV Prevalence Estimates from the Demographic and Health Surveys. Calverton: ICF International; 2012.

64. Boily M-C, Baggaley RF, Wang L, Masse B, White RG, Hayes RJ, Alary M. Heterosexual risk of HIV-1 infection per sexual act: systematic review and meta-analysis of observational studies. Lancet Infect Dis. 2009;9(2):118-29.

65. Abu-Raddad LJ, Barnabas RV, Janes H, Weiss HA, Kublin JG, Longini IMJ, Wasserheit JN, Group tHVLW. Have the explosive HIV epidemics in sub-Saharan Africa been driven by higher community viral load? AIDS. 2013;27(6):981-9. doi:10.1097/QAD.0b013e32835cb927.

66. Hargreaves JR, Davey C, White RG. Does the 'inverse equity hypothesis' explain how both poverty and wealth can be associated with HIV prevalence in sub-Saharan Africa? J Epidemiol Community Health. 2013;67(6):526-9.

67. Hargreaves J, Davey C, Fearon E, Krishnaratne S. Changes in HIV prevalence among socioeconomic groups in eight African countries: analysis of sequential cross-sectional surveys. Lancet. 2013;381:S57.

68. Wingood GM, Scd, DiClemente RJ. Application of the theory of gender and power to examine HIV-related exposures, risk factors, and effective interventions for women. Health Educ Behav. 2000;27(5):539-65.

69. Leana CR, Feldman DC. Gender differences in responses to unemployment. J Vocat Behav. 1991;38(1):65-77.

70. Sanders-Phillips K. Factors influencing HIV/AIDS in women of color. Public Health Rep. 2002;117:S151-6.

71. Wyatt GE, Riederle MH. Reconceptualizing issues that affect women's sexual decision-making and sexual functioning. Psychol Women Q. 1994;18:611-25.

72. Mkandawire-Valhmu L, Wendland C, Stevens PE, Kako PM, Dressel A, Kibicho J. Marriage as a risk factor for HIV: Learning from the experiences of HIVinfected women in Malawi. Glob Public Health. 2013;8(2):187-201.

73. Mkandawire-Valhmu L, Rodriguez R, Ammar N, Nemoto K. Surviving life as a woman: a critical ethnography of violence in the lives of female domestic workers in Malawi. Health Care Women Int. 2009;30(9):783-801. 
74. Clark S. Early marriage and HIV risks in Sub-Saharan Africa. Stud Fam Plann. 2004;35(3):149-60.

75. Asiimwe-Okiror G, Opio AA, Musinguzi J, Madraa E, Tembo G, Carael M. Change in sexual behaviour and decline in HIV infection among young pregnant women in urban Uganda. AIDS. 1997;11(14):1757-63.

76. Hallett TB, Lewis JJC, Lopman BA, Nyamukapa CA, Mushati P, Wambe M, Garnett GP, Gregson S. Age at first Sex and HIV infection in rural Zimbabwe. Stud Fam Plann. 2007;38(1):1-10.

77. Pettifor AE, Van der Straten A, Dunbar MS, Shiboski SC, Padian NS. Early age of first sex: a risk factor for HIV infection among women in Zimbabwe. AIDS 2004; 18:1435-42.

78. Stöckl H, Kalra N, Jacobi J, Watts C. Is early sexual debut a risk factor for HIV infection among women in Sub-Saharan Africa? A systematic review. Am J Reprod Immunol. 2013;69 Suppl 1:27-40.

79. Ghebremichael MS, Finkelman MD. Effect of premarital sex on sexually transmitted infections (STIS) and high risk behaviors in women. J AIDS HIV Res. 2013;5(2):59-64.

80. Conroy AA. Gender, power, and intimate partner violence: a study on couples from rural Malawi. J Interpers Violence. 2014;29(5):866-88.

81. Rivera-Rivera JN, Rodríguez Madera SL, Varas-Díaz N, Pacheco RY. The Role of Social Support on Health-Related Practices of HIV-Discordant Heterosexual Couples, vol. 24. 2014

82. Camlin CS, Kwena ZA, Dworkin SL, Cohen CR, Bukusi EA. "She mixes her business": HIV transmission and acquisition risks among female migrants in western Kenya. Soc Sci Med. 2014;102(0):146-56.

83. Hardee K, Gay J, Croce-Galis M, Peltz A. Strengthening the enabling environment for women and girls: what is the evidence in social and structural approaches in the HIV response? J Int AIDS Soc. 2014;17(1):18619.

84. Ramjee G, Daniels B. Women and HIV in Sub-Saharan Africa. AIDS Res Ther. 2013;10(1):30.

85. Ackermann L, Klerk GW. Social factors that make South African women vulnerable to HIV infection. Health Care Women Int. 2002;23(2):163-72.

86. Connolly CA, Ramjee G, Sturm AW, Abdool Karim SS. Incidence of sexually transmitted infections among HIV-positive sex workers in KwaZulu-Natal, South Africa. Sex Transm Dis. 2002;29(11):721-4.

87. Kalichman SC, Pellowski J, Turner C. Prevalence of sexually transmitted co-infections in people living with HIV/AIDS: systematic review with implications for using HIV treatments for prevention. Sex Transm Infect. 2011;87(3):183-90.

88. Wira CR, Fahey JV. A new strategy to understand how HIV infects women: identification of a window of vulnerability during the menstrual cycle. AIDS (London, England). 2008;22(15):1909-17.

89. Fortson JG. The gradient in sub-Saharan Africa: socioeconomic status and HIV/AIDS. Demography. 2008;45(2):303-22.

90. Buvé A, Lagarde E, Caraël M, Rutenberg N, Ferry B, Glynn JR, Laourou M, Akam E, Chege J, Sukwa T. Interpreting sexual behaviour data: validity issues in the multicentre study on factors determining the differential spread of HIV in four African cities. AIDS. 2001;15:S117-26.

91. Auerbach JD, Coates TJ. HIV prevention research: accomplishments and challenges for the third decade of AIDS. Am J Public Health. 2000;90(7): 1029-32.

92. Auerbach JD, Parkhurst JO, Cáceres CF. Addressing social drivers of HIV/AIDS for the long-term response: conceptual and methodological considerations. Glob Public Health. 2011;6(sup3):S293-309.

93. Coates TJ, Richter L, Caceres C. HIV Prevention 3: Behavioural strategies to reduce HIV transmission: how to make them work better. In: Lancet. 372nd ed. 2008. p. 669-84.

94. Gupta GR, Parkhurst JO, Ogden JA, Aggleton P, Mahal A. Structural approaches to HIV prevention. Lancet. 2008;372(9640):764-75.

95. Ogden J, Gupta GR, Warner A, Fisher WF. Revolutionising the AIDS response. Glob Public Health. 2011:6(sup3):S383-95.

96. Kurth AE, Celum C, Baeten JM, Vermund SH, Wasserheit JN. Combination HIV prevention: significance, challenges, and opportunities. Curr HIV/AIDS Rep. 2011;8(1):62-72.

97. Thomas-Slayter BP, Fisher WF. Social capital and AIDS-resilient communities: strengthening the AIDS response. Glob Public Health. 2011;6(Supplement 3):S323-43.

\section{Submit your next manuscript to BioMed Central and we will help you at every step:}

- We accept pre-submission inquiries

- Our selector tool helps you to find the most relevant journal

- We provide round the clock customer support

- Convenient online submission

- Thorough peer review

- Inclusion in PubMed and all major indexing services

- Maximum visibility for your research

Submit your manuscript at www.biomedcentral.com/submit 